\title{
A Procedure for Estimating the Variance of the Population Mean in Rejective Sampling
}

\author{
Marius Stefan ${ }^{1}$ and Michael A. Hidiroglou ${ }^{2}$
}

\begin{abstract}
Rejective sampling was first introduced by Hájek in 1964 as a way to select a sample consisting uniquely of distinct units. If $n$ denotes the fixed sample size, the $n$ units are drawn independently with probabilities that may vary from unit to unit and the samples in which all units are not distinct are rejected. More generally, in rejective sampling, we select repeated samples according to a basic sampling design until a selected sample meets a specified balancing tolerance. Given a set of auxiliary variables, we consider a procedure in which the probability sample is rejected unless the sample mean of the auxiliary variables is within a specified distance of its corresponding population mean. The procedure represents an alternative to the well-known balanced cube method. In this article, we propose an estimator of the variance under the rejective sampling design. We also present the results of a Monte Carlo simulation study.
\end{abstract}

Key words: Balanced sampling; rejective sampling; normality assumption.

\section{Introduction}

Auxiliary data are commonly used these days in National Statistical Offices. The resulting estimators are either regression or calibration based. The regression-based procedures are chosen so as to improve the reliability of the estimators of the parameters of interest. The calibration-based procedures ensure that the weighted totals (means) of the auxiliary data exactly agree with their population totals (means). A deficiency of these procedures is that the final weight, the product of the original design weight times a factor accounting for the auxiliary data, can be negative. One way to avoid negative weights is to use the weight bounding algorithms given in Huang and Fuller (1978) or in Deville and Särndal (1992). Another way is to use the cube method given in Deville and Tillé (2004) or the rejective procedure developed by Fuller (2009). These methods will eliminate samples in which the final weights associated with some sampled units are negative. Comparisons between the cube and the rejective procedure have been given in Legg and Yu (2010) and Chauvet et al. (2017).

In this article, we focus on the rejective procedure developed by Fuller (2009) for estimating a population mean, $\bar{Y}_{U}=N^{-1} \sum_{i \in U} y_{i}$, where $N$ is the population size and $y$ denotes a characteristic of interest. A number of procedures closely related to Fuller's (2009) rejective procedure can be found in the literature. Hájek $(1981,66)$ gives a formal definition of rejective sampling resulting from a procedure in which a Poisson sample is

${ }^{1}$ Polytehnica University of Bucarest, Splaiul Independentei nr. 313, Bucarest, RO-060042, Romania. Email: mastefan@gmail.com

2 Statistics Canada, Canada. Email: hidirog@yahoo.ca 
rejected unless it contains exactly $n$ units. The Hájek articles $(1964,1981)$ discuss the analysis of such samples extensively.

Denote as $p^{b}(s)$ the probability of selecting a specified sample $s$. The associated first order and second order inclusion probabilities are $\pi_{i}^{b}=\sum_{s \ni i} p^{b}(s)$ and $\pi_{i j}^{b}=\sum_{s \ni i, j} p^{b}(s)$, where $\pi_{i j}^{b}=\pi_{i}^{b}$ for $i=j$. A sample $s^{b}$ selected from $U$ with $\pi_{i}^{b}$ as its first order selection probabilities is a basic sample: this term was introduced by Fuller (2009). The superscript $b$ stands for basic sampling design. The expectation and variance operators under the basic sampling design will be denoted by $E_{b}(\cdot)$ and $V_{b}(\cdot)$.

We assume that a vector of auxiliary data, say $\boldsymbol{x}$, is available for each unit in the population. Let $\boldsymbol{x}_{i}^{T}=\left(x_{i 1}, \ldots, x_{i p}\right)$ be the value of $\boldsymbol{x}$ for unit $i$ in the population $U$. The known population mean is $\overline{\boldsymbol{X}}_{U}=N^{-1} \sum_{i \in U} \boldsymbol{x}_{i}$. For a given sample $s^{b}$, selected via the basic sampling design, $\hat{\bar{X}}_{H T}^{b}=N^{-1} \sum_{i \in s^{b}} \boldsymbol{x}_{i} / \pi_{i}^{b}$ is the associated Horvitz-Thompson estimator of the population mean $\overline{\boldsymbol{X}}_{U}$. Its population variance is

$$
V_{b}\left(\hat{\overline{\boldsymbol{X}}}_{H T}^{b}\right)=\frac{1}{N^{2}} \sum_{i \in U} \sum_{j \in U} \Delta_{i j}^{b} \frac{\boldsymbol{x}_{i}}{\pi_{i}^{b}} \frac{\boldsymbol{x}_{j}^{T}}{\pi_{j}^{b}}
$$

where $\Delta_{i j}^{b}=\pi_{i j}^{b}-\pi_{i}^{b} \pi_{j}^{b}$. The variance-covariance matrix $V_{b}\left(\hat{\bar{X}}_{H T}^{b}\right)$ is assumed to be invertible.

The sample mean $\hat{\boldsymbol{X}}_{H T}^{b}$ can be quite far away from the population mean $\overline{\boldsymbol{X}}_{U}$. The sample mean of samples selected with Deville and Tillé (2004)'s cube method or Fuller's (2009) rejective procedure will be "close" to the population mean.

In this article, we use Fuller's (2009) rejective procedure for selecting a sample. It is constructed based on a "distance" variable $Q$ defined as

$$
Q=\left(\hat{\overline{\boldsymbol{X}}}_{H T}^{b}-\overline{\boldsymbol{X}}_{U}\right)^{T}\left(V_{b}\left(\hat{\overline{\boldsymbol{X}}}_{H T}^{b}\right)\right)^{-1}\left(\hat{\overline{\boldsymbol{X}}}_{H T}^{b}-\overline{\boldsymbol{X}}_{U}\right) .
$$

A sample $s^{b} \subset U$ is initially selected using the basic sampling design. This sample is retained only if

$$
Q \leq \gamma^{2}
$$

where $\gamma^{2}>0$ is a pre-specified constant. If the $Q$ associated with the sample does not satisfy inequality (2), another sample $s^{b}$ is selected. The process stops when inequality (2) is satisfied, and that sample is retained. Samples $s^{b}$ that satisfy (2) will be denoted as $s^{r}$, where $r$ stands for rejective sampling. The term rejective sampling design will be associated with samples $s^{r}$, selected using rule (2). The expectation and variance operators associated with the rejective sampling design will be respectively denoted by $E_{r}(\cdot)$ and $V_{r}(\cdot)$.

Given inequality (2), the distance between the sample mean $\hat{\bar{X}}_{H T}^{b}$ and population mean $\overline{\boldsymbol{X}}_{U}$ is controlled via $\gamma^{2}$ : the smaller $\gamma^{2}$ is, the closer $\hat{\overline{\boldsymbol{X}}}_{H T}^{b}$ is to $\overline{\boldsymbol{X}}_{U}$. An approximate rejection rate can be set by suitably selecting the value of $\gamma^{2}$. High rejection rates could provide high reductions in the variance. On the other hand, low rejection rates may not reduce the variance by a large amount, but provide sufficient comfort that a very poor sample will not be selected.

A sample is balanced on a vector of auxiliary variables, $\boldsymbol{x}$, if the following equations are satisfied:

$$
\hat{\overline{\boldsymbol{X}}}_{H T}^{b}=\overline{\boldsymbol{X}}_{U}
$$


Balancing can be thought of as calibration at the design stage. Deville and Tillé (2004)'s cube method attempts to select balanced samples with pre-determined first order inclusion probabilities. Although the inclusion probabilities are exactly satisfied with the cube method, it may not be possible to satisfy equality in balancing Equation (3). Therefore, in cube sampling one has not control on the (possible) discrepancy between $\hat{\overline{\boldsymbol{X}}}_{H T}^{b}$ and $\overline{\boldsymbol{X}}_{U}$.

Given that $\gamma$ is not zero, a rejective sample $s^{r}$ selected using criterion (2) does not satisfy (3). A rejective sample $s^{r}$ will satisfy (3) only when $\gamma \rightarrow 0$, or equivalently when the rejection rate tends to $100 \%$. Therefore, a sample $s^{r}$ is only approximately balanced, but the discrepancy between $\hat{\bar{X}}_{H T}^{b}$ and $\overline{\boldsymbol{X}}_{U}$ can be controlled via the balancing tolerance $\gamma$. The drawback of rejective sampling is that the inclusion probabilities are usually unknown.

The weight associated with the basic sampling design for units belonging to a given rejective sample $s^{r}$ is

$$
w_{i, H T}^{b}=\frac{1}{\pi_{i}^{b}} .
$$

An estimator of the population mean $\bar{Y}_{U}$ that uses these weights is

$$
\hat{\bar{Y}}_{H T}^{b}=\frac{1}{N} \sum_{i \in s^{r}} w_{i, \mathrm{HT}}^{b} y_{i} .
$$

The weighted estimator $\hat{\bar{Y}}_{H T}^{b}$ based on weights $w_{i, H T}^{b}$ is not a Horvitz-Thompson estimator as it is constructed with a rejective sample $s^{r}$. Chauvet et al. (2017) pointed out that $\hat{\bar{Y}}_{H T}^{b}$ can be biased given the rejective sampling procedure. Its rejective bias is given by:

$$
B_{r}\left(\hat{Y}_{H T}^{b}\right)=\frac{1}{N} \sum_{i \in U}\left(\frac{\pi_{i}^{r}}{\pi_{i}^{b}}-1\right) y_{i}
$$

where $\pi_{i}^{r}=\Sigma_{s^{r} \ni i} p^{r}\left(s^{r}\right)$ and $p^{r}\left(s^{r}\right)$ is the probability of selecting a specified sample $s^{r}$ via the rejective sampling design. Thus, the bias can be large if some of the $\pi_{i}^{r} / \pi_{i}^{b}$ ratios are unusually large. For Poisson sampling and simple random sampling without replacement $\pi_{i}^{r} / \pi_{i}^{b}$ will be fairly close to 1 .

The unknown first order inclusion probabilities $\pi_{i}^{r}$ associated with the rejective sampling design may or may not be equal to $\pi_{i}^{b}$ for $i=1, \ldots, N$. The probability $p^{r}\left(s^{r}\right)$ of selecting a specific rejective sample $s^{r}$ can be computed exactly if $N$ and $n$ are sufficiently small to enumerate all possible samples. It is then possible to compute the inclusion probabilities $\pi_{i}^{r}$ and $\pi_{i j}^{r}=\Sigma_{s^{r} \ni i, j} p^{r}\left(s^{r}\right)$. The inclusion probabilities $\pi_{i}^{r}$ and $\pi_{i j}^{r}$ can be approximated via Monte Carlo methods if it is not possible to enumerate all samples. However, as Legg and You (2010) point out, simulating enough samples for a large population to give a precise estimate of the inclusion probability for each pair of units is impractical.

Another possibility is to approximate them. Recently, Chauvet el al. (2017) approximated the first order probability of inclusion $\pi_{i}^{r}$ via the Edgeworth expansion for a basic sampling design that used Poisson sampling. Using these approximations, an approximately unbiased estimator for the population mean can be constructed given that rejective sampling was used to select the sample. They did not, however, approximate the joint selection inclusion probabilities $\pi_{i j}^{r}$ necessary for measuring the precision of the population mean estimator under rejective sampling. 
Let $\boldsymbol{x}^{*}$ be a vector of auxiliary data available at the estimation stage. The $\boldsymbol{x}^{*}$ vector is not necessarily identical to $x$ used at the design stage in the definition of $Q$. We will suppose that $x \subseteq \boldsymbol{x}^{*}$.

There are a number of ways to use the auxiliary data $x^{*}$. The GREG regression estimator, Särndal et al. (1992, chap. 6) is the one that we chose, as it is widely used. It is given by

$$
\begin{aligned}
\hat{\bar{Y}}_{G R E G}^{b} & =\hat{\bar{Y}}_{H T}^{b}+\left(\overline{\boldsymbol{X}}_{U}^{*}-\hat{\overline{\boldsymbol{X}}}_{H T}^{* b}\right)^{T} \hat{\boldsymbol{\beta}}_{G R E G}^{b} \\
& =\frac{1}{N} \sum_{i \in s^{r}} w_{i, \mathrm{GREG}}^{b} y_{i}
\end{aligned}
$$

where

$$
\begin{aligned}
& \hat{\boldsymbol{\beta}}_{G R E G}^{b}=\left(\sum_{i \in s^{r}} \frac{\boldsymbol{x}_{i}^{*} \boldsymbol{x}_{i}^{* T}}{\pi_{i}^{b}}\right)^{-1} \sum_{i \in s^{r}} \frac{\boldsymbol{x}_{i}^{*} y_{i}}{\pi_{i}^{b}} \text { and } \\
& w_{i, G R E G}^{b}=\frac{1}{\pi_{i}^{b}}\left\{1+N\left(\overline{\boldsymbol{X}}_{U}^{*}-\hat{\boldsymbol{X}}_{H T}^{* b}\right)^{T}\left(\sum_{j \in s^{r}} \frac{\boldsymbol{x}_{j}^{*} \boldsymbol{x}_{j}^{* T}}{\pi_{j}^{b}}\right)^{-1} \boldsymbol{x}_{i}^{*}\right\}
\end{aligned}
$$

with $\hat{\overline{\boldsymbol{X}}}_{H T}^{* b}=N^{-1} \sum_{i \in s^{r}} \boldsymbol{x}_{i}^{*} / \pi_{i}^{b}$ and $\overline{\boldsymbol{X}}_{U}^{*}=N^{-1} \sum_{i \in U} \boldsymbol{x}_{i}^{*}$.

Fuller (2009) used the optimal estimator given by:

$$
\hat{\bar{Y}}_{O P T}^{b}=\overline{\boldsymbol{X}}_{U}^{* T} \hat{\boldsymbol{\beta}}_{O P T}^{b}
$$

where

$$
\hat{\boldsymbol{\beta}}_{O P T}^{b}=\left(\sum_{i \in s^{r}} \frac{\phi_{i}^{b} \boldsymbol{x}_{i}^{*} \boldsymbol{x}_{i}^{* T}}{\left(\pi_{i}^{b}\right)^{2}}\right)^{-1} \sum_{i \in s^{r}} \frac{\phi_{i}^{b} \boldsymbol{x}_{i}^{*} y_{i}}{\left(\pi_{i}^{b}\right)^{2}}
$$

and the $\phi_{i}^{b}$, s are constants determined by the design. These constants are $\phi_{i}^{b}=$ $\left(1-\pi_{i}^{b}\right), i=1, \ldots, N$ for Poisson sampling, and $\phi_{i}^{b}=\left(N_{h}-1\right)^{-1}\left(N_{h}-n_{h}\right)$ for the $i^{t h}$ element belonging to the $h^{\text {th }}$ stratum for a stratified sampling design. The estimator $\hat{\bar{Y}}_{O P T}^{b}$ is design consistent under the basic procedure, (Fuller 2009), if $\operatorname{cov}\left(\hat{\bar{X}}_{H T}^{* b T}, \hat{\bar{Y}}_{H T}^{b}\right)=O\left(n^{-1}\right)$ and if there exists a vector $c$ such that

$$
\frac{\phi_{i}^{b} x_{i}^{* T} c}{\left(\pi_{i}^{b}\right)^{2}}=\frac{1}{\pi_{i}^{b}} .
$$

Fuller (2009) proved that estimator $\hat{\bar{Y}}_{O P T}^{b}$ constructed with the rejective sample has the same limiting variance as the regression estimator that uses the first and second order inclusion probabilities associated with the basic selection procedure. Fuller et al. (2017) proposed a bootstrap procedure as an alternative way to estimate the variance of $\hat{\bar{Y}}_{O P T}^{b}$. The method, suggested for Poisson samples, also performs well with rejective Poisson samples.

We focus on estimating the variance of an estimator of $\bar{Y}_{U}$, say $\hat{\theta}$, given that rejective sampling has taken place. The variance estimator is a plug-in estimator obtained from a 
result for the rejective variance of $\hat{\theta}$ based on a normality assumption. The rejective variance estimator for $\hat{\theta}$ is expected to perform well if its distribution is normal or approximately normal. The theory will be applied to estimate the variance of $\hat{\theta}=\hat{\bar{Y}}_{H T}^{b}$ and $\hat{\theta}=\hat{\bar{Y}}_{G R E G}^{b}$ under the rejective sampling knowing that, under fairly broad regularity conditions, the limiting distributions of these estimators are normal.

The article is structured as follows. In Section 2 we obtain $V_{r}(\hat{\theta})$ assuming that the joint distribution of $\hat{\theta}$ and the vector $\hat{\bar{X}}_{H T}^{b}-\overline{\boldsymbol{X}}_{U}$ follows a multivariate normal distribution under the basic sampling design. We will also show under the normality assumption that $\hat{\theta}$ is unbiased under the rejective sampling design if $\hat{\theta}$ is unbiased under the basic sampling design. In Section 3 we show how an estimator $\hat{V}_{r}(\hat{\theta})$ of the rejective variance of $\hat{\theta}$ can be obtained. Section 4 provides the results of a simulation study that evaluates $V_{r}(\hat{\theta})$ and its estimator $\hat{V}_{r}(\hat{\theta})$. In this simulation, we focus on the weighted estimators $\hat{\bar{Y}}_{H T}^{b}$ and $\hat{\bar{Y}}_{G R E G}^{b}$ defined in Equations (5) and (7) respectively. We considered two basic procedures: simple random sampling without replacement (SRSWOR) and Bernoulli sampling without replacement (BernWOR). Finally, Section 5 contains the concluding remarks.

\section{Rejective Mean and Variance of $\hat{\boldsymbol{\theta}}$ Under the Normality Assumption}

Recall that we denoted the mean and variance of an estimator $\hat{\theta}$ of $\bar{Y}_{U}$ under the rejective sampling as $E_{r}(\hat{\theta})$ and $V_{r}(\hat{\theta})$ respectively. The population parameters $E_{r}(\hat{\theta})$ and $V_{r}(\hat{\theta})$ are based on the unknown probabilities associated with the rejective sample. However, it is possible to express them in terms of the basic sampling distribution and $Q$ given by (1). That is, $E_{r}(\hat{\theta})$ and $V_{r}(\hat{\theta})$ are set equal to the conditional mean and variance of $\hat{\theta}$ conditioned by $Q \leq \gamma^{2}$ :

$$
E_{r}(\hat{\theta})=E_{b}\left(\hat{\theta} \mid Q \leq \gamma^{2}\right) \text { and } V_{r}(\hat{\theta})=V_{b}\left(\hat{\theta} \mid Q \leq \gamma^{2}\right)
$$

We decompose the middle component of $Q$, the variance-covariance matrix $\left(V_{b}\left(\hat{\bar{X}}_{H T}^{b}\right)\right)^{-1}$, using the Cholesky decomposition. That is,

$$
\left(V_{b}\left(\hat{\overline{\boldsymbol{X}}}_{H T}^{b}\right)\right)^{-1}=\boldsymbol{P}^{T} \boldsymbol{P}
$$

where $\boldsymbol{P}^{T}$ is a $p \times p$ lower triangular matrix that is invertible.

Next, define the $p$-dimensional vector $\boldsymbol{Z}=\left(Z_{1}, \ldots, Z_{p}\right)^{T}$,

$$
\boldsymbol{Z}=\boldsymbol{P}\left(\hat{\overline{\boldsymbol{X}}}_{H T}^{b}-\overline{\boldsymbol{X}}_{U}\right)
$$

Using $\boldsymbol{Z}$, the quadratic form $Q$ given by (1) can alternatively be written as:

$$
Q=\boldsymbol{Z}^{T} \boldsymbol{Z}=\sum_{i=1}^{p} Z_{i}^{2}
$$

where $Z_{i}$ is the $i^{\text {th }}$ component of the $p$-dimensional vector $Z$.

Define the $p+1$ dimensional vector $\boldsymbol{W}$ as $\hat{\theta}$ augmented with $\boldsymbol{Z}$ : that is $\boldsymbol{W}=\left(\hat{\theta}, \boldsymbol{Z}^{T}\right)^{T}$. Using (14), the conditional mean and the conditional variance in (11) are respectively 
given by

$$
E_{r}(\hat{\theta})=E_{b}\left(\hat{\theta} \mid \boldsymbol{Z}^{T} \boldsymbol{Z} \leq \gamma^{2}\right) \text { and } V_{r}(\hat{\theta})=V_{b}\left(\hat{\theta} \mid \boldsymbol{Z}^{T} \boldsymbol{Z} \leq \gamma^{2}\right)
$$

The conditional mean and variance can be evaluated using the multivariate distribution of $\boldsymbol{W}$. We assume that the sampling distribution of $\boldsymbol{W}$ under the basic sampling design is a multivariate normal distribution of dimension $p+1$. That is $\boldsymbol{W} \sim M V N_{p+1}\left(\boldsymbol{\mu}_{w} ; \boldsymbol{\Sigma}_{w}\right)$ with $\boldsymbol{\mu}_{w}=E_{b}(\boldsymbol{W})$ and $\boldsymbol{\Sigma}_{w}=V_{b}(\boldsymbol{W})$. It follows from (13) that $E_{b}(\boldsymbol{Z})=\mathbf{0}$ and $\boldsymbol{\mu}_{w}^{T}=\left(\mu_{\theta}, \mathbf{0}^{T}\right)$, where $\mu_{\theta}=E_{b}(\hat{\theta})$.

Since the matrix $\boldsymbol{P}$ is non-singular, Equation (12) can be expressed as $\boldsymbol{P} V_{b}\left(\hat{\overline{\boldsymbol{X}}}_{H T}^{b}\right) \boldsymbol{P}^{T}=\boldsymbol{I}_{p}$ where $\boldsymbol{I}_{p}$ is the identity matrix of order $p$. It follows that the variance-covariance matrix $V_{b}(\boldsymbol{Z})$ of $\boldsymbol{Z}$ under the basic sampling design is the identity matrix $\boldsymbol{I}_{p}$. Since $\boldsymbol{Z}$ is a component of $\boldsymbol{W}$, it follows that, under the basic sampling design, $\boldsymbol{Z}$ is distributed as $\boldsymbol{Z} \sim \operatorname{MVN}_{p}\left(\mathbf{0} ; \boldsymbol{I}_{p}\right)$ and its density function is $f_{Z}(z)=(\sqrt{2 \pi})^{-p} e^{-\frac{1}{2} z^{T} z}$.

Denote as $\sigma^{2}=V_{b}(\hat{\theta})$ the variance of $\hat{\theta}$, and $\boldsymbol{\sigma}_{z \theta}=\operatorname{cov}_{b}(\boldsymbol{Z}, \hat{\theta})$ as the covariance between the random vector $\boldsymbol{Z}$ and the $\hat{\theta}$, under the basic sampling design. We have:

$$
\sigma_{z \theta}=\operatorname{cov}_{b}(\boldsymbol{Z}, \hat{\theta})=\boldsymbol{P} \operatorname{cov}_{b}\left(\hat{\overline{\boldsymbol{X}}}_{H T}^{b}, \hat{\theta}\right)
$$

where $\operatorname{cov}_{b}\left(\hat{\bar{X}}_{H T}^{b}, \hat{\theta}\right)=\left(\operatorname{cov}_{b}\left(\hat{\bar{X}}_{1 H T}^{b}, \hat{\theta}\right), \ldots, \operatorname{cov}_{b}\left(\hat{\bar{X}}_{p H T}^{b}, \hat{\theta}\right)\right)^{T}$ with $\hat{\bar{X}}_{k H T}^{b}=N^{-1} \sum_{i \in s^{b}} x_{i k} / \pi_{i}^{b}$.

The variance-covariance matrix $\boldsymbol{\Sigma}_{w}$ and its inverse are given by:

$$
\boldsymbol{\Sigma}_{w}=\left(\begin{array}{ll}
\sigma_{\theta}^{2} & \boldsymbol{\sigma}_{z \theta}^{T} \\
\boldsymbol{\sigma}_{z \theta} & \boldsymbol{I}_{p}
\end{array}\right) \text { and } \boldsymbol{\Sigma}_{w}^{-1}=\left(\begin{array}{cc}
a^{-1} & -a^{-1} \boldsymbol{\sigma}_{z \theta}^{T} \\
-a^{-1} \boldsymbol{\sigma}_{z \theta} & \boldsymbol{M}
\end{array}\right)
$$

where

$$
a=\sigma_{\theta}^{2}-\boldsymbol{\sigma}_{z \theta}^{T} \boldsymbol{\sigma}_{z \theta} \text { and } \boldsymbol{M}=\boldsymbol{I}_{p}+\frac{1}{a} \sigma_{z \theta} \boldsymbol{\sigma}_{z \theta}^{T} .
$$

The determinant of matrix $\boldsymbol{\Sigma}_{w},\left|\boldsymbol{\Sigma}_{w}\right|$, is equal to $a$.

Given the above preliminaries, we can now spell out our main result concerning the rejective mean $E_{r}(\hat{\theta})$ and the rejective variance $V_{r}(\hat{\theta})$ of estimator $\hat{\theta}$ using the normality of $\boldsymbol{W}$.

Theorem 1. Assume that the basic sampling distribution of vector $\boldsymbol{W}$ follows a multivariate normal distribution: that is $\boldsymbol{W} \sim M V N_{p+1}\left(\boldsymbol{\mu}_{w} ; \boldsymbol{\Sigma}_{w}\right)$. Given that $\hat{\overline{\boldsymbol{X}}}_{H T}^{b}$ satisfies inequality (2), the conditional mean and variance of $\hat{\theta}$ are:

$$
\text { i. } E_{r}(\hat{\theta})=E_{b}\left(\hat{\theta} \mid \boldsymbol{Z}^{T} \boldsymbol{Z} \leq \gamma^{2}\right)=\mu_{\theta}
$$

and

$$
\text { ii. } V_{r}(\hat{\theta})=V_{b}\left(\hat{\theta} \mid Z^{T} \boldsymbol{Z} \leq \gamma^{2}\right)=\sigma_{\theta}^{2}-\boldsymbol{\sigma}_{z \theta}^{T} \boldsymbol{\sigma}_{z \theta}\left(1-\frac{\int_{A} z_{1}^{2} f_{\boldsymbol{Z}}(\boldsymbol{z}) d z}{\int_{A} f_{\boldsymbol{Z}}(\boldsymbol{z}) d z}\right)
$$

where $A=\left\{\left(z_{1}, \ldots, z_{p}\right) \in \mathbb{R}^{p} \mid z_{1}^{2}+\ldots+z_{p}^{2} \leq \gamma^{2}\right\}$, and $z_{1}$ is the first component of $z^{T}=\left(z_{1}, \ldots, z_{p}\right)$. 
Proof: See Appendix A (Subsection 6.1).

Deville and Tillé (2005) used a similar normality assumption on the distribution of an augmented vector to evaluate the variance in the case of balanced sampling. Deville and Tille (2005) obtained four alternative approximations for the variance of the HorvitzThompson (Horvitz and Thompson 1952) under balanced sampling that allowed them to construct variance estimators that do not depend on second order inclusion probabilities.

It follows from part $i$. of Theorem 1 that $\hat{\theta}$ will be unbiased under the rejective sampling design if $\hat{\theta}$ is an unbiased estimator of the population mean under the basic sampling design. Part ii. of Theorem 1 provides a formula for computing the variance of $\hat{\theta}$ under the rejective sampling and the normality assumption. Notice that if the normality assumption $\boldsymbol{W} \sim M V N_{p+1}\left(\boldsymbol{\mu}_{w} ; \boldsymbol{\Sigma}_{w}\right)$ only holds approximately, then Equations (17) and (18) respectively represent approximations of $E_{r}(\hat{\theta})$ and $V_{r}(\hat{\theta})$.

Next, we show how the integrals in Equation (18) can be computed.

Proposition 1: For a positive integer $n \geq 0$, let $J_{n}(\gamma)$ the integral given by $J_{n}(\gamma)=$ $\int_{0}^{\gamma} r^{n} e^{-\frac{r^{2}}{2}} d r$

i. The integral $J_{n}(\gamma)$ obeys the following recursive relation

$$
J_{n+1}(\gamma)=n J_{n-1}(\gamma)-\gamma^{n} e^{-\frac{\gamma^{2}}{2}}, \text { where } n \geq 1 .
$$

The first two $J_{n}(\gamma)$ values are computed as: $J_{0}(\gamma)=\sqrt{2 \pi}(\Phi(\gamma)-0.5)$ and $J_{1}(\gamma)=$ $1-e^{-\frac{\gamma^{2}}{2}}$, where $\Phi(\cdot)$ is the cumulative distribution function of a standard normal distribution.

ii. Given $A$ and $f_{Z}(z)$ as defined in Theorem 1, we have that:

$$
1-\frac{\int_{A} z_{1}^{2} f_{Z}(z) d z}{\int_{A} f_{Z}(z) d z}=g(\gamma, p)
$$

where $g(\gamma, p)=\frac{\gamma^{p} e^{-\frac{\gamma^{2}}{2}}}{p J_{p-1}(\gamma)}$

Proof: See Appendix B (Subsection 6.2).

Using (12) and (15), the product $\boldsymbol{\sigma}_{z \theta}^{T} \boldsymbol{\sigma}_{z \theta}$ is given by:

$$
\boldsymbol{\sigma}_{z \theta}^{T} \boldsymbol{\sigma}_{z \theta}=\operatorname{cov}_{b}\left(\hat{\overline{\boldsymbol{X}}}_{H T}^{b}, \hat{\theta}\right)^{T}\left(V_{b}\left(\hat{\overline{\boldsymbol{X}}}_{H T}^{b}\right)\right)^{-1} \operatorname{cov}_{b}\left(\hat{\overline{\boldsymbol{X}}}_{H T}^{b}, \hat{\theta}\right) .
$$

Recall that $\sigma_{\theta}^{2}=V_{b}(\hat{\theta})$. Using (18) and (20), $V_{r}(\hat{\theta})$ can be expressed as:

$$
V_{r}(\hat{\theta})=V_{b}(\hat{\theta})-\operatorname{cov}_{b}\left(\hat{\bar{X}}_{H T}^{b}, \hat{\theta}\right)^{T}\left(V_{b}\left(\hat{\bar{X}}_{H T}^{b}\right)\right)^{-1} \operatorname{cov}_{b}\left(\hat{\bar{X}}_{H T}^{b}, \hat{\theta}\right) g(\gamma, p) .
$$

The variance matrix $V_{b}\left(\hat{\bar{X}}_{H T}^{b}\right)$ and its inverse are positive definite. The quadratic form $\boldsymbol{u}^{T}\left(V_{b}\left(\hat{\overline{\boldsymbol{X}}}_{H T}^{b}\right)\right)^{-} \boldsymbol{u}$ is greater or equal to zero for any vector $\boldsymbol{u}$. Since $g(\gamma, p)$ is positive, the second term in Equation (21) is greater or equal to zero, and it follows that $V_{r}(\hat{\theta}) \leq V_{b}(\hat{\theta})$. If an estimator $\hat{\theta}$ satisfies the conditions of Theorem 1, Equation (21) shows that its rejective variance is equal or smaller than its basic variance. 
Table 1. Values of $g(\gamma, p)$ as a function of $p$ and Rejection Rate (RR).

\begin{tabular}{|l|l|c|c|}
\hline & \multicolumn{3}{|c|}{ Rejection Rate (RR) } \\
\hline $\boldsymbol{p}$ & $\mathbf{0 . 8 0}$ & $\mathbf{0 . 9 0}$ & $\mathbf{0 . 9 5}$ \\
\hline 1 & 0.978 & 0.994 & 0.998 \\
\hline 2 & 0.892 & 0.948 & 0.974 \\
\hline 3 & 0.810 & 0.887 & 0.931 \\
\hline 4 & 0.745 & 0.830 & 0.885 \\
\hline 5 & 0.692 & 0.782 & 0.842 \\
\hline
\end{tabular}

Notice that $\lim _{\gamma \rightarrow \infty} J_{0}(\gamma)=\sqrt{\pi / 2}$ and $\lim _{\gamma \rightarrow \infty} J_{1}(\gamma)=1$. Using these limits and the recursive formula given by (19), it follows that $\lim _{\gamma \rightarrow \infty} J_{p}(\gamma)$ is finite for any fixed $p$. This implies that $\lim _{\gamma \rightarrow \infty} g(\gamma, p)=0$ and that $\lim _{\gamma \rightarrow \infty} V_{r}(\hat{\theta})=V_{b}(\hat{\theta})$. This means that when $\gamma$ is large, the basic and the rejective sampling plans are similar, and consequently there is little gain in precision in using rejective sampling.

Since $\lim _{\gamma \rightarrow 0} J_{0}(\gamma)=\lim _{\gamma \rightarrow 0} J_{1}(\gamma)=0$, it follows that $\lim _{\gamma \rightarrow 0} J_{p}(\gamma)=0$ for any fixed $p$. Hence:

$$
\lim _{\gamma \rightarrow 0} g(\gamma, p)=\lim _{\gamma \rightarrow 0} \frac{\gamma^{p}}{p J_{p-1}(\gamma)}=\lim _{\gamma \rightarrow 0} \frac{p \gamma^{p-1}}{p \gamma^{p-1} e^{-\frac{\gamma^{2}}{2}}}=1 .
$$

Using Equations (21) and (22), the minimum rejective variance of $\hat{\theta}, V_{r}^{\min }(\hat{\theta})$, is attained when $\gamma$ tends to zero. This minimum is:

$$
V_{r}^{\min }(\hat{\theta})=V_{b}(\hat{\theta})-\operatorname{cov}_{b}\left(\hat{\bar{X}}_{H T}^{b}, \hat{\theta}\right)^{T}\left(V_{b}\left(\hat{\bar{X}}_{H T}^{b}\right)\right)^{-1} \operatorname{cov}_{b}\left(\hat{\bar{X}}_{H T}^{b}, \hat{\theta}\right) .
$$

The rejective variance $V_{r}(\hat{\theta})$ is a function of $g(\gamma, p)$. Table 1 illustrates the behavior of $g(\gamma, p)$ as a function of $p$ and the rejection rate (RR). An approximate rejection rate can be set using the quantiles of the $\chi^{2}(p)$ distribution. Recall that $\gamma^{2}$ given in Equation (2) is a pre-specified constant. As $\gamma$ tends to zero, the rejection rate tends to $100 \%$ for a fixed $p$. On the other hand, for a fixed rejection rate, $\gamma$ increases as $p$ increases.

The results given in Table 1 support Equation (22). That is, for a given $p$, as the rejection rate increases, or equivalently as $\gamma$ tends to zero, $g(\gamma, p)$, tends to 1 .

For a given rejection rate, the term $g(\gamma, p)$ decreases as $p$ increases, or equivalently as $\gamma$ tends to infinity. This implies that increasing the number of variables in the distance variable $Q$ defined by (1) does not necessarily result in reductions of the rejective variance $V_{r}(\hat{\theta})$. In order to decrease $V_{r}(\hat{\theta})$, one has to make sure that the variance-covariance term $\operatorname{cov}_{b}\left(\hat{\bar{X}}_{H T}^{b}, \hat{\theta}\right)^{T}\left(V_{b}\left(\hat{\bar{X}}_{H T}^{b}\right)\right)^{-1} \operatorname{cov}_{b}\left(\hat{\bar{X}}_{H T}^{b}, \hat{\theta}\right)$ is large.

\section{Estimation of $V_{r}(\hat{\theta})$}

In this section we construct an estimator $\hat{V}_{r}(\hat{\theta})$ of the rejective variance $V_{r}(\hat{\theta})$. It is obtained by plugging into Equation (21) the estimators of its components under the basic sampling design. 
Three population parameters in Equation (21) are to be estimated. They are: i. the variance-covariance matrix $V_{b}\left(\hat{\bar{X}}_{H T}^{b}\right)$; ii. the variance $V_{b}(\hat{\theta})$; and iii. the covariance vector $\operatorname{cov}_{b}\left(\hat{\boldsymbol{X}}_{H T}^{b}, \hat{\theta}\right)$. These parameters correspond to the basic sampling design. They are estimated using inclusion probabilities $\pi_{i}^{b}$ and $\pi_{i j}^{b}$ associated with units that belong to the rejective sample $s^{r}$.

An estimator of $V_{b}\left(\hat{\overline{\boldsymbol{X}}}_{H T}^{b}\right)$ is

$$
\hat{V}_{b}\left(\hat{\overline{\boldsymbol{X}}}_{H T}^{b}\right)=\frac{1}{N^{2}} \sum_{i \in s^{\prime}} \sum_{j \in s^{r}} \frac{\Delta_{i j}^{b}}{\pi_{i j}^{b}} \frac{\boldsymbol{x}_{i}}{\pi_{i}^{b}} \frac{\boldsymbol{x}_{j}^{T}}{\pi_{j}^{b}} .
$$

Let $\hat{V}_{b}(\hat{\theta})$ and $\widehat{\operatorname{cov}}_{b}\left(\hat{\boldsymbol{X}}_{H T}^{b}, \hat{\theta}\right)$ be the respective estimators of $V_{b}(\hat{\theta})$ and $\operatorname{cov}_{b}\left(\hat{\boldsymbol{X}}_{H T}^{b}, \hat{\theta}\right)$ under the basic design. Then, an estimator for $V_{b}(\hat{\theta})$ is

$$
\hat{V}_{r}(\hat{\theta})=\hat{V}_{b}(\hat{\theta})-\widehat{\operatorname{cov}}_{b}\left(\hat{\bar{X}}_{H T}^{b}, \hat{\theta}\right)^{T}\left(\hat{V}_{b}\left(\hat{\bar{X}}_{H T}^{b}\right)\right)^{-1} \widehat{\operatorname{cov}}_{b}\left(\hat{\bar{X}}_{H T}^{b}, \hat{\theta}\right) g(\gamma, p)
$$

Remark 1: The plug-in estimator $\hat{V}_{r}(\hat{\theta})$ is obtained by replacing the unknown parameters $V_{b}(\hat{\theta}), \operatorname{cov}_{b}\left(\hat{\bar{X}}_{H T}^{b}, \hat{\theta}\right)$ and $V_{b}\left(\hat{\bar{X}}_{H T}^{b}\right)$ by their respective estimators $\hat{V}_{b}(\hat{\theta})$, $\widehat{\operatorname{cov}}_{b}\left(\hat{\bar{X}}_{H T}^{b}, \hat{\theta}\right)$ and $\hat{V}_{b}\left(\hat{\overline{\boldsymbol{X}}}_{H T}^{b}\right)$. They are unbiased under the basic procedure. That is, if one was to average their values over the set of samples $s^{b}$, their expectation would be $V_{b}(\hat{\theta})$, $\operatorname{cov}_{b}\left(\hat{\overline{\boldsymbol{X}}}_{H T}^{b}, \hat{\theta}\right)$ and $V_{b}\left(\hat{\overline{\boldsymbol{X}}}_{H T}^{b}\right)$ respectively. However, given that we compute their values with the rejective samples $s^{r}$, some bias may occur.

Remark 2: Part i. of Theorem 1 can be applied to each of the three estimators, $\hat{V}_{b}(\hat{\theta})$, $\widehat{\operatorname{cov}}_{b}\left(\hat{\boldsymbol{X}}_{H T}^{b}, \hat{\theta}\right)$ and $\hat{V}_{b}\left(\hat{\boldsymbol{X}}_{H T}^{b}\right)$. If their basic sampling distribution is approximately normal, they will be approximately unbiased when they are computed using the $s^{r}$ samples. This in turn will provide an estimator $\hat{V}_{r}(\hat{\theta})$ of $V_{r}(\hat{\theta})$ that will be approximately unbiased. Results in Section 4 illustrate that the bias of $\hat{V}_{r}(\hat{\theta})$ decreases as $n$ increases.

\section{Simulation Study}

In this section we report on a Monte Carlo simulation that evaluates the Equation (21) for the rejective variance $V_{r}(\hat{\theta})$ and its estimator $\hat{V}_{r}(\hat{\theta})$, when $\hat{\theta}=\hat{\bar{Y}}_{H T}^{b}$ and $\hat{\theta}=\hat{\bar{Y}}_{G R E G}^{b}$. We considered two basic sampling designs with equal probabilities: one of fixed sample size and the other one with random sample size. These two basic sampling designs are:

1. SRSWOR of fixed sample size $n$ with inclusion probabilities $\pi_{i}^{b}=n / N$, and

2. BernWOR of mean sample size $n$ with equal inclusion probabilities $\pi_{i}^{b}=n / N$.

Next, we describe how the population variances and their estimators are obtained for the two estimators $\hat{\bar{Y}}_{H T}^{b}$ and $\hat{\bar{Y}}_{G R E G}^{b}$ and the two sampling designs. We denote by $V_{h b}\left(\hat{\bar{X}}_{H T}^{b}\right)$, $h=1,2$ the variance-covariance matrix of $\hat{\overline{\boldsymbol{X}}}_{H T}^{b}=N^{-1} \sum_{i \in s^{b}} \boldsymbol{x}_{i} / \pi_{i}^{b}$ under the basic sampling design. The subscript $h$ is set to 1 when SRSWOR is used as the basic sampling design. It is set to 2 when BernWOR is used as the basic sampling design. For SRSWOR, the matrix $V_{1 b}\left(\hat{\bar{X}}_{H T}^{b}\right)$ is $(1-f) n^{-1}(N-1)^{-1} \sum_{i \in U}\left(\boldsymbol{x}_{i}-\overline{\boldsymbol{X}}_{U}\right)\left(\boldsymbol{x}_{i}-\overline{\boldsymbol{X}}_{U}\right)^{T}$. For BernWOR, $V_{2 b}\left(\hat{\overline{\boldsymbol{X}}}_{H T}^{b}\right)$ is $(1-f) n^{-1} N^{-1} \sum_{i \in U} \boldsymbol{x}_{i} \boldsymbol{x}_{i}^{T}$, where $f=n / N$ (see Särndal et al. 1992, Result 5.4.1., 170). 
The estimated population mean $\hat{\bar{X}}_{H T}^{b}$ was based on the two-dimensional vector $\boldsymbol{x}_{k}=$ $\left(x_{1 k}, x_{2 k}\right)^{T}$ for SRSWOR, to ensure that the matrix $V_{1 b}\left(\hat{\bar{X}}_{H T}^{b}\right)$ would be non-singular. For BernWOR, $\hat{\boldsymbol{X}}_{H T}^{b}$ was based on the three-dimensional vector $\boldsymbol{x}_{k}=\left(1, x_{1 k}, x_{2 k}\right)^{T}$. The threedimensional vector of auxiliary variables $\boldsymbol{x}_{k}^{*}=\left(1, x_{1 k}, x_{2 k}\right)^{T}$ was used to construct $\hat{\bar{Y}}_{G R E G}^{b}$ for both SRSWOR and BernWOR.

We computed the population variance $V_{b}(\hat{\theta})$ and the population covariance $\operatorname{cov}_{b}\left(\hat{\bar{X}}_{H T}^{b}, \hat{\theta}\right)$ for the two basic sampling designs, SRSWOR with fixed sample size $n$ and BernWOR with expected sample size $n$. We denote them as $V_{h b}(\hat{\theta})$ and $\operatorname{cov}_{h b}\left(\hat{\boldsymbol{X}}_{H T}^{b}, \hat{\theta}\right)$ for $h=1,2$, where $\hat{\theta}$ is either $\hat{\bar{Y}}_{H T}^{b}=N^{-1} \sum_{i \in s^{r}} y_{i} / \pi_{i}^{b}$ or $\hat{\bar{Y}}_{G R E G}^{b}=\overline{\bar{Y}}_{H T}^{b}+$ $\left(\overline{\boldsymbol{X}}_{U}^{*}-\hat{\boldsymbol{X}}_{H T}^{* b}\right)^{T} \hat{\boldsymbol{\beta}}_{G R E G}^{b}$. Given that the population data, $y$ and $x^{*}$ are known, it is possible to compute an exact population variance for $\hat{\bar{Y}}_{H T}^{b}$ (see Särndal et al. 1992, Result 2.8.1., 43) and an exact population covariance $\operatorname{cov}_{b}\left(\hat{\overline{\boldsymbol{X}}}_{H T}^{b}, \hat{\bar{Y}}_{H T}^{b}\right)$ (see Särndal et al. 1992, Result 5.4.1., 170). On the other hand, this is not possible for the regression estimator $\hat{\bar{Y}}_{G R E G}^{b}$. Särndal et al. (1992, Result 6.6.1., 235) provide an approximate population variance for $\hat{\bar{Y}}_{G R E G}^{b}$ based on a Taylor linearization of $\hat{\bar{Y}}_{G R E G}^{b}$. The Taylor expansion can also be used to approximate $\operatorname{cov}_{h b}\left(\hat{\bar{X}}_{H T}^{b}, \hat{\bar{Y}}_{G R E G}^{b}\right)$. However, these approximations are only reasonable for moderate to large values of $n$.

Given this drawback, we chose to compute Monte Carlo values for $V_{h b}\left(\hat{\bar{Y}}_{G R E G}^{b}\right)$ and $\operatorname{cov}_{h b}\left(\hat{\bar{X}}_{H T}^{b}, \hat{\bar{Y}}_{G R E G}^{b}\right)$ by sampling a large number of basic samples $s^{b}$ from the population $U$. Although we could have computed $V_{h b}\left(\hat{\bar{Y}}_{H T}^{b}\right)$ and $\operatorname{cov}_{h b}\left(\hat{\bar{X}}_{H T}^{b}, \hat{\bar{Y}}_{H T}^{b}\right)$ exactly, we evaluated them using the large number of samples $s^{b}$ selected from the population.

The estimators $\hat{V}_{h b}\left(\hat{\bar{X}}_{H T}^{b}\right), \hat{V}_{h b}(\hat{\theta})$ and $\widehat{\operatorname{cov}}_{h b}\left(\hat{\bar{X}}_{H T}^{b}, \hat{\theta}\right)$ were obtained via the standard theory in Särndal et al. (1992) for the Horvitz-Thompson and GREG estimators. They were computed using the data of the selected rejective samples $s^{r}$ and the inclusion probabilities inherited from the basic sample design. Estimators $\hat{V}_{h b}\left(\hat{\bar{Y}}_{H T}^{b}\right)$ are based on Result 2.8.1. (Särndal et al. 1992, 43). Estimators $\hat{V}_{h b}\left(\hat{\overline{\boldsymbol{X}}}_{H T}^{b}\right)$ and $\widehat{\operatorname{cov}}_{h b}\left(\hat{\overline{\boldsymbol{X}}}_{H T}^{b}, \hat{\bar{Y}}_{H T}^{b}\right)$ are based on Result 5.4.1. (Särndal et al. 1992, 170). For the GREG estimator, $\hat{V}_{h b}\left(\hat{\bar{Y}}_{G R E G}^{b}\right)$ and $\widehat{\operatorname{cov}}_{h b}\left(\hat{\bar{X}}_{H T}^{b}, \hat{\bar{Y}}_{G R E G}^{b}\right)$ use Result 6.6.1. (Särndal et al. 1992, 235).

We now explain how the simulation was carried out. The dependent variable $y$ was generated using the following linear model:

$$
y_{k}=1+x_{1 k}+5 x_{2 k}+e_{k}, k=1, \ldots, N \text { with } e_{k} \sim N(0 ; 1)
$$

where $N=1,000$. The population of $y$-values generated by (26) has mean $\bar{Y}_{U}=21.147$ and variance $S_{y U}^{2}=234.72$. The population coefficient of determination associated with model (26) is $R^{2}=99.5 \%$. The values of $x_{1}$ are generated using a normal distribution of mean 10 and variance 1 . The values of $x_{2}$ are generated using a gamma distribution of mean 2 and variance 10. The differential mix of distributions for generating the independent variables $x$ was chosen to illustrate how well Theorem 1 held under nonnormal, asymmetric distributions.

We carried out two separate simulations. The objective of the first simulation was to compute accurately the population variance $V_{h b}(\hat{\theta})$ and covariance $\operatorname{cov}_{h b}\left(\hat{\bar{X}}_{H T}^{b}, \hat{\theta}\right)$ for $h=1,2$. We selected $L=500,000$ basic samples, $s^{b}$, from the population $U$ for each of the sampling procedures, SRSWOR $(h=1)$ and BernWOR $(h=2)$. For the $l^{\text {th }}$ selected sample, we computed $\hat{\theta}^{(l)}$, where $\hat{\theta}^{(l)}=\hat{\bar{Y}}_{H T}^{b(l)}$ or $\hat{\theta}^{(l)}=\hat{\bar{Y}}_{G R E G}^{b(l)}$. The resulting Monte Carlo 
variances and covariances are given by

$$
V_{h b}^{M C 1}(\hat{\theta})=\frac{1}{L} \sum_{l=1}^{L}\left(\hat{\theta}^{(l)}-E_{h b}^{M C 1}\left(\hat{\theta}^{(l)}\right)\right)^{2}
$$

and

$$
\operatorname{cov}_{h b}^{M C 1}\left(\hat{\overline{\boldsymbol{X}}}_{H T}^{b}, \hat{\theta}\right)=\frac{1}{L} \sum_{l=1}^{L}\left(\hat{\overline{\boldsymbol{X}}}_{H T}^{b(l)}-E_{h b}^{M C 1}\left(\hat{\overline{\boldsymbol{X}}}_{H T}^{b}\right)\right)\left(\hat{\boldsymbol{\theta}}^{(l)}-E_{h b}^{M C 1}(\hat{\theta})\right)
$$

where $E_{h b}^{M C 1}(\hat{\theta})=\sum_{l=1}^{L} \hat{\boldsymbol{\theta}}^{(l)} / L$ and $E_{h b}^{M C 1}\left(\hat{\boldsymbol{X}}_{H T}^{b}\right)=\sum_{l=1}^{L} \hat{\boldsymbol{X}}_{H T}^{b(l)} / L$ with $\hat{\boldsymbol{\theta}}^{(l)}$ and $\hat{\boldsymbol{X}}_{H T}^{b(l)}$ the respective values of $\hat{\theta}$ and $\hat{\boldsymbol{X}}_{H T}^{b}$ for the $l^{\text {th }}$ selected basic sample $s^{b}$.

The values of the rejective variance $V_{r}(\hat{\theta})$ are computed via Equation (21) that depends on $V_{h b}^{M C 1}\left(\hat{\bar{X}}_{H T}^{b}\right), V_{h b}^{M C 1}(\hat{\theta})$ and $\operatorname{cov}_{h b}^{M C 1}\left(\hat{\overline{\boldsymbol{X}}}_{H T}^{b}, \hat{\theta}\right)$. These variances are denoted as $V_{1 r}(\hat{\theta})$ for SRSWOR and $V_{2 r}(\hat{\theta})$ for BernWOR.

The objective of the second simulation was to test how accurate the rejective variance $V_{h r}(\hat{\theta})$ based on (21) and its estimator $\hat{V}_{h r}(\hat{\theta})$ were under the rejective sampling. The $\gamma^{2}$ value was chosen so as to yield rejective samples with a $90 \%$ rejection rate for both SRSWOR and BernWOR. The $\gamma^{2}$ value was chosen by trial and error for both of these sampling designs. For SRSWOR, a value of $\gamma^{2}$ equal to $0.05\left(\gamma_{1}^{2}=0.05\right)$ resulted in a rejection rate of $90 \%$ of the basic samples $s^{b}$. The $\gamma^{2}$ had to be increased to 0.22 $\left(\gamma_{2}^{2}=0.22\right)$ for BernWOR to obtain the $90 \%$ rejection rate. Noting that $Q$ is approximately distributed as a $\chi^{2}(p)$ where $p$ is the length of vector $\boldsymbol{x}_{k}$, we could have obtained the value of $\gamma^{2}$ using the quantiles of $\chi^{2}(p)$. However, the trial and error approach proved to be better in getting exact $90 \%$ rejection rates.

Given the required $\gamma^{2}$ for each of the basic sampling procedures, SRSWOR and BernWOR, we selected $L=500,000$ rejective samples $s^{r}$ from a large number of basic samples $s^{b}$, based on criterion (2). For each selected rejective sample $s^{r}$, we computed $\hat{V}_{h b}\left(\hat{\bar{X}}_{H T}^{b}\right), \hat{V}_{h b}(\hat{\theta})$ and $\widehat{\operatorname{cov}}_{h b}\left(\hat{\bar{X}}_{H T}^{b}, \hat{\theta}\right)$, for $h=1,2$. The estimated values of $V_{r}(\hat{\theta})$, denoted by $\hat{V}_{1 r}(\hat{\theta})$ for SRSWOR and by $\hat{V}_{2 r}(\hat{\theta})$ for BernWOR, were obtained using Equation (25) of Section 3.

For the $\ell^{\text {th }}$ selected rejective sample let:

- $\hat{\theta}^{(\ell)}$ be the value of estimator $\hat{\theta}$ for the $\ell^{\text {th }}$ sample,

- $\hat{V}_{h r}(\hat{\theta})^{(\ell)}$ be the value of estimator $\hat{V}_{h r}(\hat{\theta}), h=1,2$ for the $\ell^{\text {th }}$ sample, and

- $\hat{V}_{h b}(\hat{\theta})^{(\ell)}$ be the value of estimator $\hat{V}_{h b}(\hat{\theta}), h=1,2$ for the $\ell^{\text {th }}$ sample.

For $h=1,2$ the Monte Carlo expectation and the Monte Carlo variance of estimator $\hat{\theta}$ are computed as:

$$
E_{h r}^{M C 2}(\hat{\theta})=\frac{1}{L} \sum_{\ell=1}^{L} \hat{\theta}^{(\ell)} \text { and } V_{h r}^{M C 2}(\hat{\theta})=\frac{1}{L} \sum_{\ell=1}^{L}\left(\hat{\theta}^{(\ell)}-E_{h r}^{M C 2}(\hat{\theta})\right)^{2} .
$$

The Monte Carlo expectation of estimators $\hat{V}_{h r}(\hat{\theta})$ and $\hat{V}_{h b}(\hat{\theta})$ are computed as:

$$
E_{h r}^{M C 2}\left(\hat{V}_{h r}(\hat{\theta})\right)=\frac{1}{L} \sum_{\ell=1}^{L} \hat{V}_{h r}(\hat{\theta})^{(\ell)} \text { and } E_{h r}^{M C 2}\left(\hat{V}_{h b}(\hat{\theta})\right)=\frac{1}{L} \sum_{\ell=1}^{L} \hat{V}_{h b}(\hat{\theta})^{(\ell)}
$$




\subsection{Rejective Sampling Using SRSWOR}

The following observations can be made from Table 2 . The estimators $\hat{\bar{Y}}_{H T}^{b}$ and $\hat{\bar{Y}}_{G R E G}^{b}$ are virtually unbiased under the rejective sampling design. This follows because the Monte Carlo expectation of $\hat{\bar{Y}}_{H T}^{b}$ and $\hat{\bar{Y}}_{G R E G}^{b}$, represented by $E_{1 r}^{M C 2}(\hat{\theta})$, is quite close to the true population mean $\bar{Y}_{U}=21.147$, for all values of $n$.

We compare the various combinations of population variances $V_{1 b}^{M C 1}(\hat{\theta}), V_{1 r}^{M C 2}(\hat{\theta})$ and $V_{1 r}(\hat{\theta})$.

The use of rejective sampling results in gains in terms of population variance. This follows by comparing the variance $V_{1 b}^{M C 1}(\hat{\theta})$ under SRSWOR to the Monte Carlo variance $V_{1 r}^{M C 2}(\hat{\theta})$ under rejective sampling. The gains are quite large for the $\hat{\bar{Y}}_{H T}^{b}$ estimator: this makes sense, as we have drawn samples whose mean, $\hat{\bar{X}}_{H T}^{b}$, is quite close to the population mean $\hat{\bar{X}}_{U}$. On the other hand, for the regression estimator $\hat{\bar{Y}}_{G R E G}^{b}$, the gains are not as large since it uses auxiliary data that are well correlated with $y$.

The value of $V_{1 r}(\hat{\theta})$ is compared to the Monte Carlo variance of $\hat{\theta}$ under rejective sampling, $V_{1 r}^{M C 2}(\hat{\theta})$. Recall that the two components of $V_{1 r}(\hat{\theta})$, defined by Equation (21), were obtained via simulation under SRSWOR. The value of $V_{1 r}(\hat{\theta})$ is quite close to $V_{1 r}^{M C 2}(\hat{\theta})$ for $\hat{\theta}=\hat{\bar{Y}}_{H T}^{b}$. For $\hat{\theta}=\hat{\bar{Y}}_{G R E G}^{b}$, the largest difference between $V_{1 r}\left(\hat{\bar{Y}}_{G R E G}^{b}\right)$ and $V_{1 r}^{M C 2}\left(\hat{\bar{Y}}_{G R E G}^{b}\right)$ occurs when $n=10$ : in this case we have that $V_{1 r}\left(\hat{\bar{Y}}_{G R E G}^{b}\right)=0.1792$ and $V_{1 r}^{M C 2}$ $\left(\hat{\bar{Y}}_{G R E G}^{b}\right)=0.0947$. This means that for $n=10$, the normality assumption of Theorem 1 is far from being satisfied. Theorem 1 is not applicable for small values of $n$. For moderate to large values of $n, V_{1 r}\left(\hat{\bar{Y}}_{G R E G}^{b}\right)$ and $V_{1 r}^{M C 2}\left(\hat{\bar{Y}}_{G R E G}^{b}\right)$ are approximately equal.

The covariance in the second term of $V_{1 r}\left(\hat{\bar{Y}}_{G R E G}^{b}\right)$ given by Equation (21) can be approximated using Result 6.6.1. (Särndal et al. 1992, 235). That is $\operatorname{cov}_{1 b}\left(\hat{\bar{X}}_{H T}^{b}, \hat{\bar{Y}}_{G R E G}^{b}\right) \approx$ $(1-f) n^{-1} \boldsymbol{S}_{x E} \quad$ where $\quad \boldsymbol{S}_{x E}=(N-1)^{-1}\left(\sum_{i \in U} E_{i} \boldsymbol{x}_{i}-N \bar{E} \overline{\boldsymbol{X}}_{U}\right), \quad \boldsymbol{\beta}_{0, G R E G}=\left(\sum_{i \in U}\right.$ $\left.\boldsymbol{x}_{i}^{*} \boldsymbol{x}_{i}^{* T}\right)^{-1} \sum_{i \in U} \boldsymbol{x}_{i}^{*} y_{i}, E_{i}=y_{i}-\boldsymbol{x}_{i}^{* T} \boldsymbol{\beta}_{0, G R E G}$ and $\bar{E}=N^{-1} \sum_{i \in U} E_{i}$. It can be shown that $\sum_{i \in U} E_{i} \boldsymbol{x}_{i}=0$ and $\sum_{i \in U} E_{i}=0$, using the system of equations $\sum_{i \in U}\left(y_{i}-\right.$ $\left.\boldsymbol{x}_{i}^{* T} \boldsymbol{\beta}_{0, G R E G}\right) \boldsymbol{x}_{i}^{*}=0$ and $\boldsymbol{x}_{i} \subset \boldsymbol{x}_{i}^{*}$. Hence, $\boldsymbol{S}_{x E}=0$ and the second term in Equation (21) is approximately zero. This explains why the variances $V_{1 b}^{M C 1}\left(\hat{\bar{Y}}_{G R E G}^{b}\right)$ and $V_{1 r}\left(\hat{\bar{Y}}_{G R E G}^{b}\right)$ are approximately equal for all values of $n$ considered in Table 2 . On the other hand, we noticed that $V_{1 r}\left(\hat{\bar{Y}}_{G R E G}^{b}\right)$ and $V_{1 r}^{M C 2}\left(\hat{\bar{Y}}_{G R E G}^{b}\right)$ are getting closer as $n$ increases. Consequently, the rejective variance of $\hat{\bar{Y}}_{G R E G}^{b}$ tends to its basic variance if SRSWOR is the basic sampling design. This is not the case for $\hat{\bar{Y}}_{H T}^{b}$, as Table 2 clearly illustrates.

The estimator $\hat{V}_{1 b}(\hat{\theta})$ is unbiased for $V_{1 b}(\hat{\theta})$ under the SRSWOR basic sampling design. However, when its Monte Carlo mean is computed over the set of the rejective samples $s^{r}$, this estimator has some bias. This is readily observed from Table 2 by comparing the value of $E_{1 r}^{M C 2}\left[\hat{V}_{1 b}(\hat{\theta})\right]$ to the variance $V_{1 b}^{M C 1}(\hat{\theta})$. For $n=10$ the bias is large for both estimators, $\hat{\theta}=\hat{\bar{Y}}_{H T}^{b}$ and $\hat{\theta}=\hat{\bar{Y}}_{G R E G}^{b}$. Under the rejective sampling, as $n$ increases, the bias of $\hat{V}_{1 b}(\hat{\theta})$ as an estimator of $V_{1 b}(\hat{\theta})$ decreases (see Remark 2). A similar conclusion holds for $\widehat{\operatorname{cov}}_{1 b}\left(\hat{\bar{X}}_{H T}^{b}, \hat{\theta}\right)$ as an estimator of $\operatorname{cov}_{1 b}\left(\hat{\bar{X}}_{H T}^{b}, \hat{\theta}\right)$ under rejective sampling (numerical results not shown).

We next turn to the estimators of the rejective variance of $\hat{\theta}$. The variance of $\hat{\theta}$ under rejective sampling can be estimated by $\hat{V}_{1 b}(\hat{\theta})$ or by $\hat{V}_{1 r}(\hat{\theta})$. The first estimator, $\hat{V}_{1 b}(\hat{\theta})$, ignores that rejective sampling has taken place. The second estimator, $\hat{V}_{1 r}(\hat{\theta})$, obtained via estimators $\hat{V}_{1 b}\left(\hat{\boldsymbol{X}}_{H T}^{b}\right), \hat{V}_{1 b}(\hat{\theta})$ and $\widehat{\operatorname{cov}}_{1 b}\left(\hat{\boldsymbol{X}}_{H T}^{b}, \hat{\theta}\right)$, accounts for the rejective sampling. 


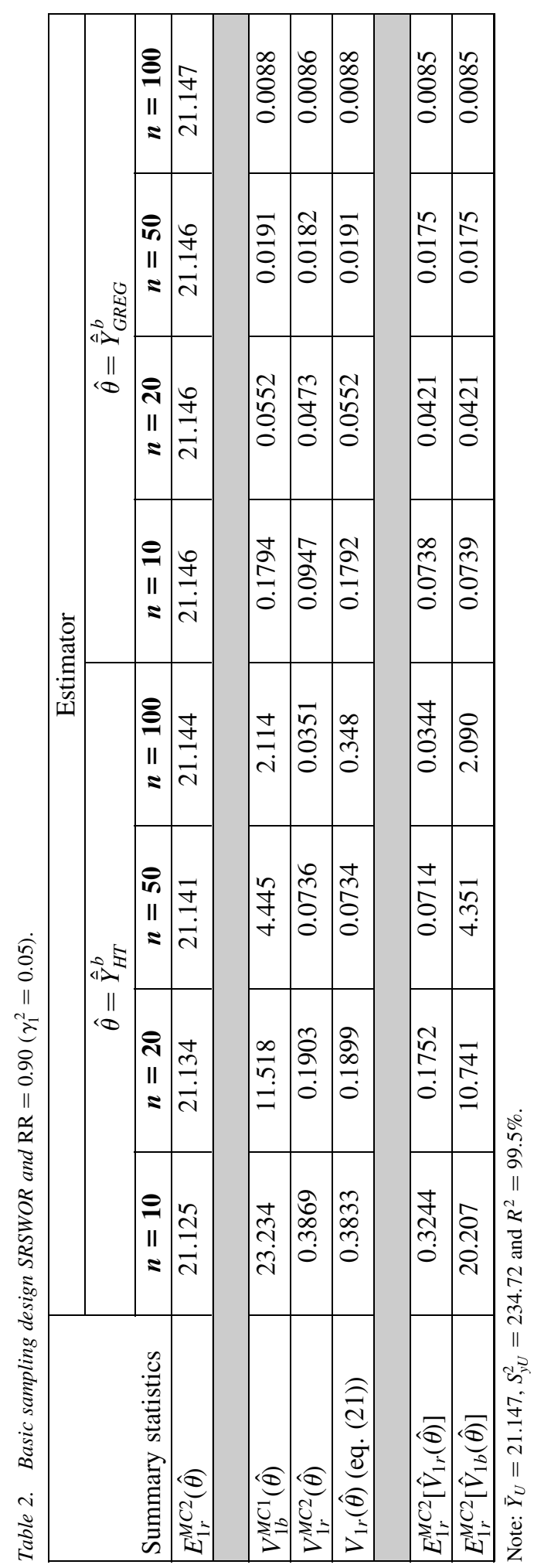


The estimated variance of $\hat{\bar{Y}}_{H T}^{b}$ can be computed as $\hat{V}_{1 b}\left(\hat{\bar{Y}}_{H T}^{b}\right)$ or as $\hat{V}_{1 r}\left(\hat{\bar{Y}}_{H T}^{b}\right)$. Given that the population variance of $\hat{\bar{Y}}_{H T}^{b}$ is $V_{1 r}^{M C 2}(\hat{\theta}), \hat{V}_{1 b}\left(\hat{\bar{Y}}_{H T}^{b}\right)$ is highly biased for all values of $n$. On the other hand, $\hat{V}_{1 r}\left(\hat{Y}_{H T}^{b}\right)$ has a small bias since, under rejective sampling, estimators $\hat{V}_{1 b}\left(\hat{\overline{\boldsymbol{X}}}_{H T}^{b}\right), \hat{V}_{1 b}(\hat{\theta})$ and $\widehat{\operatorname{cov}}_{1 b}\left(\hat{\bar{X}}_{H T}^{b}, \hat{\theta}\right)$ are biased for $V_{1 b}\left(\hat{\bar{X}}_{H T}^{b}\right), V_{1 b}(\hat{\theta})$ and $\operatorname{cov}_{1 b}\left(\hat{\bar{X}}_{H T}^{b}, \hat{\theta}\right)$ respectively (see Remark 1 ). As $n$ increases, the bias of these estimators tends to zero. It follows that estimator $\hat{V}_{1 r}\left(\hat{\bar{Y}}_{H T}^{b}\right)$ becomes unbiased for the rejective variance of $\hat{\bar{Y}}_{H T}^{b}$ as $n$ becomes large.

There are two alternative estimators for estimating the variance of $\hat{\bar{Y}}_{G R E G}^{b}$ under the rejective sampling:

i. $\hat{V}_{1 b}\left(\hat{\bar{Y}}_{G R E G}^{b}\right)$ based on the standard theory and probabilities $\left(\pi_{i}^{b}, \pi_{i j}^{b}\right)$ of the basic sampling procedure and

ii. $\hat{V}_{1 r}\left(\hat{\bar{Y}}_{G R E G}^{b}\right)$ obtained using Equation (25).

From a bias point of view, they are very similar if we compare $E_{1 r}^{M C 2}\left[\hat{V}_{1 b}\left(\hat{\bar{Y}}_{G R E G}^{b}\right)\right]$ to $E_{1 r}^{M C 2}\left[\hat{V}_{1 r}\left(\hat{\bar{Y}}_{G R E G}^{b}\right)\right]$. This result is not surprising. We noticed that for the GREG estimator, its rejective and basic variances are getting closer. On the other hand, under rejective sampling, the bias of $\hat{V}_{1 b}\left(\hat{\bar{Y}}_{G R E G}^{b}\right)$ as an estimator of $V_{1 b}\left(\hat{\bar{Y}}_{G R E G}^{b}\right)$ tends to zero. It follows that the bias of $\hat{V}_{1 b}\left(\hat{\bar{Y}}_{G R E G}^{b}\right)$ as an estimator of $V_{1 r}\left(\hat{\bar{Y}}_{G R E G}^{b}\right)$ decreases as $n$ becomes larger. Fuller (2009) proved that this would be the case for the optimal estimator, $\hat{\bar{Y}}_{O P T}^{b}$, defined in Equation (9).

A referee pointed out that the proposed variance estimator in (25) shows large bias for small sample sizes in the simulation study. He suggested that we consider alternative variance estimators proposed in Deville and Tillé (2005). These estimators, that we denote as DT, were developed for the variance of the Horvitz-Thompson estimator $\hat{\bar{Y}}_{H T}^{b}$ in the context of balanced sampling defined by Equation (3).

Using Deville and Tillé (2005)'s notation, the DT variance estimators are given by

$$
\hat{V}_{r}^{D T i}(\hat{\theta})=\frac{1}{N^{2}} \sum_{k \in s^{r}} c_{k i}\left(\breve{y}_{k}-\hat{y}_{k}^{*}\right)^{2}, \quad i=1, \ldots, 5
$$

where $\hat{\breve{y}}_{k}^{*}=\hat{\vec{x}}_{k}^{T}\left(\sum_{l \in s^{r}} c_{l i} \breve{\boldsymbol{x}}_{l} \breve{\boldsymbol{x}}_{l}^{T}\right)^{-1} \sum_{l \in s^{r}} c_{l i} \breve{\boldsymbol{x}}_{l} \breve{y}_{l}$, with $\breve{\boldsymbol{x}}_{k}=\boldsymbol{x}_{k} / \pi_{k}$ and $\breve{y}_{k}=y_{k} / \pi_{k}$.

Deville and Tillé (2005) proposed five $c_{k}$ 's labeled as $c_{k i}, i=1, \ldots, 5$. The $c_{k}$ 's are:

i. $\quad c_{k 1}=1-\pi_{k}$

ii. $\quad c_{k 2}=\left(1-\pi_{k}\right) \frac{n}{n-p}$ where $p$ is the length of vector $\boldsymbol{x}_{k}$

iii. $\quad c_{k 3}=\left(1-\pi_{k}\right) \frac{\sum_{k \in s^{r}}\left(1-\pi_{k}\right)}{\sum_{k \in s^{r}} D_{k k 1}}$ where $D_{k k 1}=c_{k 1}-c_{k 1}^{2} \breve{x}_{k}^{T}\left(\sum_{l \in s^{r}} c_{l 1} \breve{\boldsymbol{x}}_{l} \breve{\boldsymbol{x}}_{l}^{T}\right)^{-1} \breve{\boldsymbol{x}}_{k}$

iv. $\quad c_{k 4}=\frac{b_{k 4}}{\pi_{k}} \frac{n}{n-p} \frac{N-p}{N}$ where $b_{k 4}$ are solutions to the nonlinear equations

$$
\pi_{k}\left(1-\pi_{k}\right)=b_{k 4}-b_{k 4}^{2} \breve{\boldsymbol{x}}_{k}^{T}\left(\sum_{l \in U} b_{l 4} \breve{\boldsymbol{x}}_{l} \breve{\boldsymbol{x}}_{l}^{T}\right)^{-1} \breve{\boldsymbol{x}}_{k}, k=1, \ldots, N
$$

and

v. $c_{k 5}$ where the $c_{k 5}$ 's are solutions to the nonlinear equations 


$$
1-\pi_{k}=c_{k 5}-c_{k 5}^{2} \breve{x}_{k}^{T}\left(\sum_{l \in s^{r}} c_{l 5} \breve{\boldsymbol{x}}_{l} \breve{\boldsymbol{x}}_{l}^{T}\right)^{-1} \breve{\boldsymbol{x}}_{k}, k=1, \ldots, n
$$

We followed Matei and Tillé (2005) to solve the nonlinear equations by the fixed point technique using a single iteration.

We denote our estimator given by (25) as $\hat{V}_{r}^{S H}(\hat{\theta})$. We analyzed the performance of the DT variance estimators, and compared them to our own, for $\hat{\theta}=\hat{\bar{Y}}_{H T}^{b}$, via a third simulation study using SRSWOR as the basic sampling design. Since under SRSWOR, $\hat{V}_{r}^{D T 2}(\hat{\theta})=\hat{V}_{r}^{D T 3}(\hat{\theta})$, we computed $\hat{V}_{r}^{D T i}(\hat{\theta})$ for $i=1,2,4,5$. We used $\boldsymbol{x}_{k}=\left(x_{1 k}, x_{2 k}\right)$, implying that $p=2$.

We generated a large number $(L=500,000)$ of rejective samples $s^{r}$ to compute the Monte Carlo rejective variance as:

$$
V_{r}^{M C 3}(\hat{\theta})=\frac{1}{L} \sum_{l=1}^{L}\left(\hat{\theta}^{(l)}-\hat{\bar{\theta}}\right)^{2}
$$

with $\hat{\bar{\theta}}=L^{-1} \sum_{l=1}^{L} \hat{\theta}^{(l)}$ where $\hat{\theta}^{(l)}$ is the $\ell^{\text {th }}$ value of $\hat{\theta}$.

Let $\hat{V}_{r}$ be one of the five variance estimators $\left(\hat{V}_{r}^{S H}(\hat{\theta})\right.$ and $\left.\hat{V}_{r}^{D T i}(\hat{\theta}), i=1,2,4,5\right)$ to be compared in the simulation study, and $\hat{V}_{r}^{(l)}$ its $\ell^{\text {th }}$ value. The variance estimators are compared via the Relative Bias (RB):

$$
R B\left(\hat{V}_{r}\right)=\frac{E_{r}^{M C 3}\left(\hat{V}_{r}\right)}{V_{r}^{M C 3}(\hat{\theta})}-1
$$

where the Monte Carlo mean of $\hat{V}_{r}$ is computed as $E_{r}^{M C 3}\left(\hat{V}_{r}\right)=L^{-1} \sum_{l=1}^{L} \hat{V}_{r}^{(l)}$.

We considered two more values for the rejection rate: 0.99 and 0.997 . When the basic sampling design is SRSWOR, a rejection rate of $99 \%$ is obtained for $\gamma_{3}^{2}=0.003$ whereas a rejection rate of $99.7 \%$ is obtained for $\gamma_{4}^{2}=0.0009$. The results of the relative bias of the five variance estimators under the three rejective rates, 0.90, 0.99 and 0.997 , are given

\begin{tabular}{|c|c|c|c|c|c|c|}
\hline & & $\hat{V}_{r}^{S H}(\hat{\theta})$ & $\hat{V}_{r}^{D T 1}(\hat{\theta})$ & $\hat{V}_{r}^{D T 2}(\hat{\theta})$ & $\hat{V}_{r}^{D T 4}(\hat{\theta})$ & $\hat{V}_{r}^{D T 5}(\hat{\theta})$ \\
\hline \multirow{4}{*}{$\begin{array}{c}\mathbf{R R}=\mathbf{0 . 9 0} \\
\left(\gamma_{1}^{2}=0.05\right)\end{array}$} & $n=10$ & -16.1 & -79.9 & -74.9 & -75.0 & -76.0 \\
\hline & $n=20$ & -7.9 & -77.5 & -75.0 & -75.0 & -75.4 \\
\hline & $n=50$ & -2.9 & -75.9 & -74.9 & -74.9 & -75.0 \\
\hline & $n=100$ & -1.9 & -75.4 & -74.9 & -74.9 & -74.9 \\
\hline \multirow{4}{*}{$\begin{array}{c}\mathbf{R R}=\mathbf{0 . 9 9} \\
\left(\gamma_{3}^{2}=0.003\right)\end{array}$} & $n=10$ & -20.6 & -31.5 & -14.3 & -14.4 & -17.9 \\
\hline & $n=20$ & -10.6 & -23.6 & -15.1 & -15.1 & -16.4 \\
\hline & $n=50$ & -3.5 & -17.7 & -14.3 & -14.3 & -14.6 \\
\hline & $n=100$ & -1.6 & -16.1 & -14.4 & -14.4 & -14.5 \\
\hline \multirow{4}{*}{$\begin{array}{c}\mathbf{R R}=\mathbf{0 . 9 9 7} \\
\left(\gamma_{4}^{2}=0.0009\right)\end{array}$} & $n=10$ & -22.0 & -23.7 & -4.6 & -4.7 & -8.6 \\
\hline & $n=20$ & -11.3 & -14.7 & -5.2 & -5.2 & -6.6 \\
\hline & $n=50$ & -5.3 & -9.3 & -5.5 & -5.6 & -5.9 \\
\hline & $n=100$ & -2.7 & -6.9 & -5.0 & -5.0 & -5.1 \\
\hline
\end{tabular}
in Table 3.

Table 3. Relative bias (\%) of the variance estimators for $\hat{\theta}=\hat{\bar{Y}}_{H T}^{b}$ : basic sampling design SRSWOR.

Note: $\bar{Y}_{U}=21.147, S_{y U}^{2}=234.72$ and $R^{2}=99.5 \%$. 
The relative bias of the four DT variance estimators differ across rejection rates and sample sizes. The relative bias of these estimators decreases as the rejection rate increases. When the rejection rate is $90 \%$, none of the DT variance estimators have a relative bias that is smaller than the SH variance estimator. All four DT variance estimators display very similar relative bias, ranging from $-79.9 \%$ to $-74.9 \%$ for all sample sizes considered.

The relative bias of the DT estimators decreases when the rejection rate is increased to 99\%. DT2, DT4 and DT5 have relative biases that are similar. Their bias is smaller than the one associated with SH only for $n=10$. DT1 has relative bias that is consistently higher than the one associated with $\mathrm{SH}$.

The DT estimators have the smallest relative bias when the rejection rate is $99.7 \%$. This is not surprising as the balancing Equation (3) is closely satisfied at such a high rejection rate. Once more, DT1 has the largest relative bias amongst the DT variance estimators. The other DT variance estimators have smaller relative bias than the SH estimator when $n$ is 10 or 20 . For $n$ equal to 50 or 100 , the $\mathrm{SH}$ estimator has the smallest relative bias.

\subsection{Rejective Sampling Using BernWOR}

In Table 4, we present the results obtained when the basic procedure is BernWOR and the rejection rate is $90 \%$. The results in the table are with respect to the expected sample size.

The Monte Carlo expectation of $\hat{\theta}=\hat{\bar{Y}}_{H T}^{b}$ and $\hat{\theta}=\hat{\bar{Y}}_{G R E G}^{b}$ represented by $E_{2 r}^{M C 2}(\hat{\theta})$ is quite close to the true population mean $\bar{Y}_{U}=21.147$, for all values of $n$.

BernWOR adds extra variation because the sample size is random. For the HT estimator, the basic variance $V_{2 b}^{M C 1}\left(\hat{Y}_{H T}^{b}\right)$ under BernWOR is approximately three times larger than $V_{1 b}^{M C 1}\left(\hat{\bar{Y}}_{H T}^{b}\right)$ under SRSWOR. For the GREG estimator, $V_{2 b}^{M C 1}\left(\hat{\bar{Y}}_{G R E G}^{b}\right)$ and $V_{1 b}^{M C 1}\left(\hat{Y}_{G R E G}^{b}\right)$ are much closer except for $n=10$. In this case, $V_{2 b}^{M C 1}\left(\hat{Y}_{G R E G}^{b}\right)$ is 0.3098 as opposed to 0.1794 for $V_{1 b}^{M C 1}\left(\hat{\bar{Y}}_{G R E G}^{b}\right)$. Due to the small expected sample size $(n=10)$, the value $V_{2 b}^{M C 1}\left(\hat{\bar{Y}}_{G R E G}^{b}\right)=0.3098$ was computed using BernWOR samples $s^{b}$ of size larger than 5. There is still a large difference between 0.3098 and 0.1794 compared to the corresponding differences associated to larger expected sample sizes considered in the simulation. This shows that it is fairly inappropriate to use a GREG estimator in samples with very few observations when the GREG estimator is based on a three-dimensional vector $\left(1, x_{1 \mathrm{k}}, x_{2 k}\right)^{T}$.

The gains in terms of variance due to the rejective sampling design are larger for SRSWOR as opposed to BernWOR for $\hat{\theta}=\hat{\bar{Y}}_{H T}^{b}$. This is observed by comparing the ratios $V_{1 b}^{M C 1}\left(\hat{\bar{Y}}_{H T}^{b}\right) / V_{1 r}^{M C 2}\left(\hat{\bar{Y}}_{H T}^{b}\right)$ in Table 2 for SRSWOR to the ratios $V_{2 b}^{M C 1}\left(\hat{\bar{Y}}_{H T}^{b}\right) / V_{2 r}^{M C 2}\left(\hat{\bar{Y}}_{H T}^{b}\right)$ in Table 4 for BernWOR. The ratio $V_{1 b}^{M C 1}\left(\hat{\bar{Y}}_{H T}^{b}\right) / V_{1 r}^{M C 2}\left(\hat{\bar{Y}}_{H T}^{b}\right)$ is approximately equal to 60 , whereas the ratio $V_{2 b}^{M C 1}\left(\hat{\bar{Y}}_{H T}^{b}\right) / V_{2 r}^{M C 2}\left(\hat{\bar{Y}}_{H T}^{b}\right)$ is approximately equal to 20 for any sample size in the simulation. For $\hat{\theta}=\hat{\bar{Y}}_{G R E G}^{b}$, the gains in terms of variance are similar for SRSWOR and BernWOR.

Note that $V_{2 r}(\hat{\theta})$ and $V_{2 r}^{M C 2}(\hat{\theta})$, for $\hat{\theta}=\hat{\bar{Y}}_{H T}^{b}$ and $\hat{\theta}=\hat{\bar{Y}}_{G R E G}^{b}$, are getting closer as $n$ increases.

For $\hat{\bar{Y}}_{G R E G}^{b}$, its rejective and basic variances are getting closer as $n$ increases. This observation is in line with what happens when SRSWOR is the initial sampling design. A similar argument can be used to support this observation for moderate to large values of $n$. The population covariance $\operatorname{cov}_{2 b}\left(\hat{\bar{X}}_{H T}^{b}, \hat{\bar{Y}}_{G R E G}^{b}\right)$ is approximately equal to 


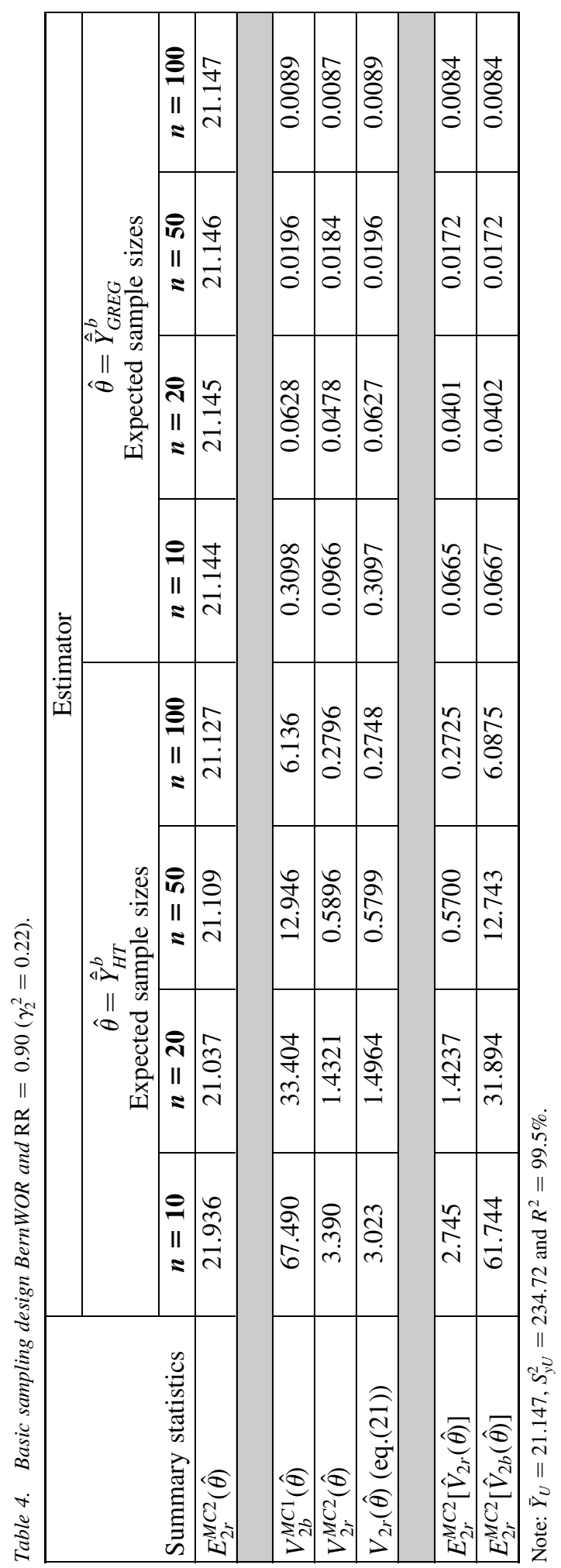


$(1-f) n^{-1} N^{-1} \sum_{i \in U} E_{i} \boldsymbol{x}_{i}$. This approximation is obtained by applying Result 6.6.1., in Särndal et al. $(1992,235)$ to the case of the covariance between the GREG and the HT estimators when the sampling design is BernWOR. Since $\sum_{i \in U} E_{i} \boldsymbol{x}_{i}=0$, it follows that the second term in Equation (21) approaches zero as $n$ increases.

The population variance $V_{2 r}(\hat{\theta})$ is estimated by $\hat{V}_{2 r}(\hat{\theta})$. Comparing the Monte Carlo mean of the resulting estimator $E_{2 r}^{M C 2}\left[\hat{V}_{2 r}(\hat{\theta})\right]$ to the Monte Carlo variance $V_{2 r}^{M C 2}(\hat{\theta})$, we see that large differences occur for $n=10$ for both $\hat{\bar{Y}}_{H T}^{b}$ and $\hat{\bar{Y}}_{G R E G}^{b}$. As $n$ increases the bias of these estimators tends to zero.

\section{Conclusion}

The use of rejective sampling brings about reductions in variance for both the HorvitzThompson estimator and the GREG regression estimator. The reduction is more significant for the Horvitz-Thompson than for the regression estimator.

We obtained an exact formula for the rejective variance $V_{r}(\hat{\theta})$ of a population mean estimator $\hat{\theta}$ by assuming that under the basic sampling design, $\hat{\theta}$ and its associated auxiliary data mean $\hat{\bar{X}}_{H T}^{b}$, have a joint normal distribution. This result was obtained by conditioning on $Q \leq \gamma^{2}$. This allowed us to avoid computing (approximating) the unknown rejective inclusion probabilities $\pi_{i}^{r}$ and $\pi_{i j}^{r}$.

If the normal distribution is only approximate, $V_{r}(\hat{\theta})$ represents an approximation of the true rejective variance. There are estimators $\hat{\theta}$ of the population mean $\bar{Y}_{U}$ whose distribution tends to normality. Our result is applicable to such estimators in samples with sufficiently large $n$.

An estimator $\hat{V}_{r}(\hat{\theta})$ for the rejective variance was obtained by replacing the unknown parameters in $V_{r}(\hat{\theta})$ by estimators associated with the basic sampling design and based on the rejective sample.

A simulation study was undertaken to evaluate the accuracy of $V_{r}(\hat{\theta})$ and the bias of its estimator $\hat{V}_{r}(\hat{\theta})$. This was carried out for two estimators $\left(\hat{\theta}=\hat{\bar{Y}}_{H T}^{b}\right.$ and $\left.\hat{\theta}=\hat{\bar{Y}}_{G R E G}^{b}\right)$ and two basic sampling designs (SRSWOR and BernWOR). The empirical results show that if the normality assumption is approximately respected, the formula for $V_{r}(\hat{\theta})$ performs well for moderate to large values of $n$. For small sample sizes, the proposed variance estimator $\hat{V}_{r}(\hat{\theta})$ is biased but the bias reduces as $n$ increases.

\section{Appendix}

\subsection{Appendix A: Proof of Theorem 1}

i. Denote by $f_{\boldsymbol{W}}(\boldsymbol{w})$ the density function of vector $\boldsymbol{W}$ where $\boldsymbol{w}=(x, z)$ and $z=\left(z_{1}, \ldots, z_{p}\right)$. The marginal density of $\boldsymbol{Z}$ is

$$
f_{\boldsymbol{Z}}(z)=\int_{-\infty}^{\infty} f_{W}(x, z) d x=\frac{1}{(\sqrt{2 \pi})^{p}} e^{-\frac{z^{T} z}{2}}
$$

Using the formula for the inverse of matrix $\boldsymbol{\Sigma}_{W}$, the determinant $\left|\boldsymbol{\Sigma}_{w}\right|=a$ and the definition of matrix $\boldsymbol{M}, \boldsymbol{M}=\boldsymbol{I}_{p}+a^{-1} \boldsymbol{\sigma}_{z \theta} \boldsymbol{\sigma}_{z \theta}^{T}$, the density function $f_{\boldsymbol{W}}(\boldsymbol{w})$ of vector $\boldsymbol{W}$ is: 


$$
\begin{aligned}
f_{W}(\boldsymbol{w}) & =\frac{1}{(\sqrt{2 \pi})^{p+1} \sqrt{\left|\boldsymbol{\Sigma}_{w}\right|}} e^{-\frac{1}{2}\left(\boldsymbol{w}-\boldsymbol{\mu}_{w}\right)^{T} \boldsymbol{\Sigma}_{w}^{-1}\left(\boldsymbol{w}-\boldsymbol{\mu}_{w}\right)} \\
& =\frac{1}{\sqrt{2 \pi a}} e^{-\frac{1}{2 a}\left[\left(x-\mu_{\theta}\right)-z^{T} \sigma_{z \theta}\right]^{2}} f_{\boldsymbol{Z}}(\boldsymbol{z})
\end{aligned}
$$

The conditional mean $E_{b}\left(\hat{\theta} \mid \boldsymbol{Z}^{T} \boldsymbol{Z} \leq \gamma^{2}\right)$ can alternatively be rewritten as $E_{b}(\hat{\theta} \mid \boldsymbol{Z} \in \boldsymbol{A})$, where $\boldsymbol{A}$ is the random event defined as $\boldsymbol{A}=\left\{\omega \mid Z_{1}(\omega)^{2}+\ldots+Z_{p}(\omega)^{2} \leq \gamma^{2}\right\}$. In order to compute $E_{b}\left(\hat{\theta} \mid \boldsymbol{Z}^{T} \boldsymbol{Z} \leq \gamma^{2}\right)$ and $E_{b}\left(\hat{\theta}^{2} \mid \boldsymbol{Z}^{T} \boldsymbol{Z} \leq \gamma^{2}\right)$ one needs to find the density function $f_{\hat{\theta} \mid \mathbf{Z} \in \boldsymbol{A}}(x)$ of the conditional variable $\hat{\theta} \mid \boldsymbol{Z} \in \boldsymbol{A}$.

The cumulative probability function of $\hat{\theta} \mid \boldsymbol{Z} \in \boldsymbol{A}$ is given by:

$$
F_{\hat{\theta} \mid \mathbf{Z} \in \boldsymbol{A}}(x)=P(\hat{\theta} \leq x \mid \boldsymbol{Z} \in \boldsymbol{A})=\frac{P(\hat{\theta} \leq x \cap \boldsymbol{Z} \in \boldsymbol{A})}{P(\boldsymbol{Z} \in \boldsymbol{A})}=\frac{\int_{-\infty}^{x} \int_{A} f_{\boldsymbol{W}}(t, \boldsymbol{z}) d z d t}{\int_{A} f_{\boldsymbol{Z}}(\boldsymbol{z}) d \boldsymbol{z}}
$$

where $A$ is the set defined in Theorem 1 .

The conditional density function $f_{\hat{\theta} \mid \mathbf{Z} \in \boldsymbol{A}}(x)$ is obtained by differentiating $F_{\hat{\theta} \mid \mathbf{Z} \in \boldsymbol{A}}(x)$ with respect to $x$ :

$$
f_{\hat{\theta} \mid \mathbf{Z} \in \boldsymbol{A}}(x)=\frac{d F_{\hat{\theta} \mid \mathbf{Z} \in \boldsymbol{A}}(x)}{d x}=\frac{\int_{A} f_{W}(x, z) d z}{\int_{A} f_{Z}(z) d z}
$$

As a consequence, the conditional mean $E_{b}\left(\hat{\theta} \mid Z^{T} \boldsymbol{Z} \leq \gamma^{2}\right)$ can be computed as:

$$
E_{b}\left(\hat{\theta} \mid Z^{T} \boldsymbol{Z} \leq \gamma^{2}\right)=\frac{\int_{-\infty}^{\infty} x\left(\int_{A} f_{W}(x, z) d z\right) d x}{\int_{A} f_{\boldsymbol{Z}}(\boldsymbol{z}) d z}=\frac{\int_{A}\left(\int_{-\infty}^{\infty} x f_{\boldsymbol{W}}(x, \boldsymbol{z}) d x\right) d z}{\int_{A} f_{\boldsymbol{Z}}(\boldsymbol{z}) d z}
$$

The integral $\int_{-\infty}^{\infty} x f_{W}(x, z) d x$ in (A2) can be computed as follows:

$$
\begin{aligned}
\int_{-\infty}^{\infty} x f_{W}(x, z) d x & =\int_{-\infty}^{\infty}\left(x-\mu_{\theta}\right) f_{W}(x, z) d x+\mu_{\theta} \int_{-\infty}^{\infty} f_{W}(x, z) d x \\
& =\int_{-\infty}^{\infty}\left(x-\mu_{\theta}\right) f_{W}(x, z) d x+\mu_{\theta} f_{Z}(z)
\end{aligned}
$$

The first term is obtained by replacing $f_{w}(x, z)$ given in (A1):

$$
\begin{aligned}
\int_{-\infty}^{\infty}\left(x-\mu_{\theta}\right) f_{W}(x, z) d x & =\frac{1}{\sqrt{2 \pi a}} \int_{-\infty}^{\infty}\left(x-\mu_{\theta}\right) e^{-\frac{1}{2 a}\left[\left(x-\mu_{\theta}\right)-z^{T} \boldsymbol{\sigma}_{z \theta}\right]^{2}} d x f_{\boldsymbol{Z}}(z) \\
& =\left(z^{T} \boldsymbol{\sigma}_{z \theta}\right) f_{\mathbf{Z}}(\boldsymbol{z})
\end{aligned}
$$


In the last equation we used the value of integral:

$$
\frac{1}{\sqrt{2 \pi a}} \int_{-\infty}^{\infty} y e^{-\frac{1}{2 a}\left(y-z^{T} \sigma_{z \theta}\right)^{2}} d y=z^{T} \boldsymbol{\sigma}_{z \theta}
$$

Hence,

$$
\int_{-\infty}^{\infty} x f_{W}(x, z) d x=\left(z^{T} \boldsymbol{\sigma}_{z \theta}+\mu_{\theta}\right) f_{Z}(z)
$$

Replacing (A3) in (A2), we obtain:

$$
E_{b}\left(\hat{\theta} \mid \boldsymbol{Z}^{T} \boldsymbol{Z} \leq \gamma^{2}\right)=\frac{\int_{A}\left(z^{T} \boldsymbol{\sigma}_{z \theta}+\mu_{\theta}\right) f_{\boldsymbol{Z}}(z) d z}{\int_{A} f_{\boldsymbol{Z}}(\boldsymbol{z}) d z}=\frac{\int_{A}\left(z^{T} \boldsymbol{\sigma}_{z \theta}\right) f_{\boldsymbol{Z}}(\boldsymbol{z}) d z}{\int_{A} f_{\boldsymbol{Z}}(z) d z}+\mu_{\theta}=\mu_{\theta}
$$

In (A4) we use that $\int_{A} z_{i} f_{\boldsymbol{Z}}(z) d z=0$ which implies $\int_{A}\left(z^{T} \boldsymbol{\sigma}_{z \theta}\right) f_{\mathbf{Z}}(z) d z=0$ (see (B7) below). ii A similar argument holds for $E_{b}\left(\hat{\theta}^{2} \mid \boldsymbol{Z}^{T} \boldsymbol{Z} \leq \gamma^{2}\right)$ :

$$
E_{b}\left(\hat{\theta}^{2} \mid \boldsymbol{Z}^{T} \boldsymbol{Z} \leq \gamma^{2}\right)=\frac{\int_{-\infty}^{\infty} x^{2}\left(\int_{A} f_{W}(x, z) d z\right) d x}{\int_{A} f_{\boldsymbol{Z}}(\boldsymbol{z}) d z}=\frac{\int_{A}\left(\int_{-\infty}^{\infty} x^{2} f_{W}(x, \boldsymbol{z}) d x\right) d z}{\int_{A} f_{\boldsymbol{Z}}(\boldsymbol{z}) d \boldsymbol{z}}
$$

The numerator of (A5) can be computed as

$$
\begin{aligned}
\int_{-\infty}^{\infty} x^{2} f_{W}(x, z) d x= & \int_{-\infty}^{\infty}\left(x-\mu_{\theta}\right)^{2} f_{W}(x, z) d x+2 \mu_{\theta} \int_{-\infty}^{\infty}\left(x-\mu_{\theta}\right) f_{W}(x, z) d x \\
& +\mu_{\theta}^{2} \int_{-\infty}^{\infty} f_{W}(x, z) d x
\end{aligned}
$$

We now compute each term of (A6). For the first term we replace the density function $f_{W}(x, z)$ by Equation (A1):

$$
\begin{aligned}
\int_{-\infty}^{\infty}\left(x-\mu_{\theta}\right)^{2} f_{W}(x, z) d x & =\frac{1}{\sqrt{2 \pi a}} \int_{-\infty}^{\infty}\left(x-\mu_{\theta}\right)^{2} e^{-\frac{1}{2 a}\left[\left(x-\mu_{\theta}\right)-z^{T} \sigma_{z \theta}\right]^{2}} d x f_{\boldsymbol{Z}}(\boldsymbol{z}) \\
& =\left[a+\left(\boldsymbol{z}^{T} \boldsymbol{\sigma}_{z \theta}\right)^{2}\right] f_{\boldsymbol{Z}}(\boldsymbol{z})
\end{aligned}
$$

In the above equation we used the value of integral

$$
\frac{1}{\sqrt{2 \pi a}} \int_{-\infty}^{\infty} y^{2} e^{-\frac{1}{2 a}\left[y-z^{T} \sigma_{z \theta}\right)^{2}} d y=a+\left(z^{T} \boldsymbol{\sigma}_{z \theta}\right)^{2}
$$


For the second term of (A6) we use (A3):

$$
2 \mu_{\theta} \int_{-\infty}^{\infty}\left(x-\mu_{\theta}\right) f_{W}(x, z) d x=2 \mu_{\theta}\left(z^{T} \sigma_{z \theta}\right) f_{Z}(z)
$$

The third term in (A6) is

$$
\mu_{\theta}^{2} \int_{-\infty}^{\infty} f_{W}(x, z) d x=\mu_{\theta}^{2} f_{Z}(z)
$$

Replacing (A7), (A8) and (A9) into (A6) one obtains:

$$
\int_{-\infty}^{\infty} x^{2} f_{W}(x, z) d x=\left[a+\left(z^{T} \boldsymbol{\sigma}_{z \theta}\right)^{2}+2 \mu_{\theta}\left(z^{T} \boldsymbol{\sigma}_{z \theta}\right)+\mu_{\theta}^{2}\right] f_{\boldsymbol{Z}}(\boldsymbol{z})
$$

Replacing (A10) into (A5) and using again $\int_{A}\left(z^{T} \boldsymbol{\sigma}_{z \theta}\right) f_{Z}(z) d z=0$, we obtain:

$$
E_{b}\left(\hat{\theta}^{2} \mid \boldsymbol{Z}^{T} \boldsymbol{Z} \leq \gamma^{2}\right)=a+\mu_{\theta}^{2}+\frac{\int_{A}\left(z^{T} \boldsymbol{\sigma}_{z \theta}\right)^{2} f_{\boldsymbol{Z}}(\boldsymbol{z}) d z}{\int_{A} f_{\boldsymbol{Z}}(\boldsymbol{z}) d z} .
$$

The conditional variance follows from (A4) and (A11):

$$
V_{b}\left(\hat{\theta} \mid \boldsymbol{Z}^{T} \boldsymbol{Z} \leq \gamma^{2}\right)=a+\frac{\int_{A}\left(z^{T} \boldsymbol{\sigma}_{z \theta}\right)^{2} f_{\boldsymbol{Z}}(\boldsymbol{z}) d z}{\int_{A} f_{\boldsymbol{Z}}(\boldsymbol{z}) d \boldsymbol{z}}
$$

Now, by symmetry, $\int_{A} z_{i}^{2} f_{\boldsymbol{Z}}(z) d z=\int_{A} z_{1}^{2} f_{\boldsymbol{Z}}(z) d z$ and $\int_{A} z_{i} z_{j} f_{\boldsymbol{Z}}(z) d z=0$ for $i \neq j$ (see (B8) below). It follows that:

$$
\int_{A}\left(z^{T} \boldsymbol{\sigma}_{z \theta}\right)^{2} f_{\boldsymbol{Z}}(z) d z=\left(\boldsymbol{\sigma}_{z \theta}^{T} \boldsymbol{\sigma}_{z \theta}\right) \int_{A} z_{1}^{2} f_{\boldsymbol{Z}}(z) d z
$$

Using (A12), (A13) and the definition of $a, a=\sigma_{\theta}^{2}-\boldsymbol{\sigma}_{z \theta}^{T} \boldsymbol{\sigma}_{z \theta}$, the conditional variance is obtained:

$$
V_{b}\left(\hat{\theta} \mid \boldsymbol{Z}^{T} \boldsymbol{Z} \leq \gamma^{2}\right)=\sigma_{\theta}^{2}-\boldsymbol{\sigma}_{z \theta}^{T} \boldsymbol{\sigma}_{z \theta}\left(1-\frac{\int_{A} z_{1}^{2} f_{\boldsymbol{Z}}(\boldsymbol{z}) d \boldsymbol{z}}{\int_{A} f_{\boldsymbol{Z}}(\boldsymbol{z}) d \boldsymbol{z}}\right)
$$

and this proves ii.

\subsection{Appendix B: Proof of Proposition 1}

i. We have that:

$$
J_{n+1}(\gamma)=\int_{0}^{\gamma} r^{n}\left(-e^{-\frac{r^{2}}{2}}\right)^{\prime} d r=\left.r^{n} e^{-\frac{r^{2}}{2}}\right|_{\gamma} ^{0}+n \int_{0}^{\gamma} r^{n-1} e^{-\frac{r^{2}}{2}} d r=n J_{n-1}(\gamma)-\gamma^{n} e^{-\frac{\gamma^{2}}{2}}
$$

and relation (19) is proved. 
The first two " $J$ " terms are:

$$
\begin{aligned}
& J_{0}(\gamma)=\frac{\sqrt{2 \pi}}{\sqrt{2 \pi}} \int_{0}^{\gamma} e^{-\frac{r^{2}}{2}} d r=\sqrt{2 \pi}(\Phi(\gamma)-\Phi(0))=\sqrt{2 \pi}(\Phi(\gamma)-0.5), \\
& J_{1}(\gamma)=\int_{0}^{\gamma}\left(-e^{-\frac{r^{2}}{2}}\right)^{\prime} d r=\left.e^{-\frac{r^{2}}{2}}\right|_{\gamma} ^{0}=1-e^{-\frac{\gamma^{2}}{2}}
\end{aligned}
$$

ii. We use the transformation to $p$-spherical coordinates. For a point $z \in A$ with $z=\left(z_{1}, \ldots, z_{p}\right)$, its $p$-spherical coordinates are $\left(r, \theta_{1}, \ldots, \theta_{p-1}\right)$, where:

$$
\begin{aligned}
& z_{1}=r \cos \theta_{1} \\
& z_{2}=r \sin \theta_{1} \cos \theta_{2} \\
& z_{3}=r \sin \theta_{1} \sin \theta_{2} \cos \theta_{3} \\
& \vdots \\
& z_{p-1}=r \sin \theta_{1} \sin \theta_{2} \ldots \sin \theta_{p-2} \cos \theta_{p-1} \\
& z_{p}=r \sin \theta_{1} \sin \theta_{2} \ldots \sin \theta_{p-2} \sin \theta_{p-1}
\end{aligned}
$$

with $r \in[0, \gamma], \theta_{1} \in[0, \pi], \ldots, \theta_{p-2} \in[0, \pi]$ and $\theta_{p-1} \in[0,2 \pi]$. Using (B1), we have that $z^{T} z=r^{2}$.

Let us denote by $B$ the set defined as the Cartesian product

$$
B=[0, \gamma] \times[0, \pi] \times \ldots \times[0, \pi] \times[0,2 \pi]
$$

The transformation (B1) maps the set $A$ into the set B. The Jacobian of the transformation (B1) is given by:

$$
d z_{1} d z_{2} \ldots d z_{p}=r^{p-1} \sin ^{p-2} \theta_{1} \sin ^{p-3} \theta_{2} \ldots \sin \theta_{p-2} d r d \theta_{1} \ldots d \theta_{p-2} d \theta_{p-1}
$$

For a positive integer $n \geq 0$, let us denote by $T_{n}$ the integral

$$
T_{n}=\int_{0}^{\pi} \sin ^{n} \theta d \theta
$$

It can be shown that $T_{n}$ obeys the following recursive relationship:

$$
n T_{n}=(n-1) T_{n-2}
$$


Using (B1) and (B2), the integrals $\int_{A} f_{Z}(z) d z$ and $\int_{A} z_{1}^{2} f_{Z}(z) d z$ are given by:

$$
\begin{gathered}
\int_{A} f_{Z}(z) d z=\frac{1}{(\sqrt{2 \pi})^{p}} \int_{B} e^{-\frac{1}{2} r^{2}} r^{p-1} \sin ^{p-2} \theta_{1} \sin ^{p-3} \theta_{2} \ldots \sin \theta_{p-2} d r d \theta_{1} \ldots d \theta_{p-2} d \theta_{p-1} \Rightarrow \\
\int_{A} f_{Z}(z) d z=\frac{2 \pi}{(\sqrt{2 \pi})^{p}} J_{p-1}(\gamma) T_{p-2} T_{p-3} \ldots T_{1}
\end{gathered}
$$

and

$$
\begin{gathered}
\int_{A} z_{1}^{2} f_{Z}(z) d z=\frac{1}{(\sqrt{2 \pi})^{p}} \int_{B} e^{-\frac{1}{2} r^{2}} r^{p+1} \sin ^{p-2} \theta_{1} \cos ^{2} \theta_{1} \sin ^{p-3} \theta_{2} \ldots \sin \theta_{p-2} d r d \theta_{1} \ldots d \theta_{p-1} \Rightarrow \\
\int_{A} z_{1}^{2} f_{Z}(z) d z=\frac{2 \pi}{(\sqrt{2 \pi})^{p}} J_{p+1}(\gamma)\left(T_{p-2}-T_{p}\right) T_{p-3} \ldots T_{1}
\end{gathered}
$$

Using (B3), one gets that $\left(T_{p-2}-T_{p}\right)=T_{p-2} / p$ and replacing in (B5) it follows that

$$
\int_{A} z_{1}^{2} f_{Z}(z) d z=\frac{2 \pi}{p(\sqrt{2 \pi})^{p}} J_{p+1}(\gamma) T_{p-2} T_{p-3} \ldots T_{1}
$$

From (B4) and (B6) it results that

$$
1-\frac{\int_{A} z_{1}^{2} f_{Z}(z) d z}{\int_{A} f_{Z}(z) d z}=1-\frac{J_{p+1}(\gamma)}{p J_{p-1}(\gamma)}
$$

Then, the recursive formula (19) obtained for $J_{n}(\gamma)$ is used and Equation (20) follows.

In Appendix A, we stated without proof that $\int_{A} z_{1} f_{\boldsymbol{Z}}(z) d z$ and $\int_{A} z_{1} z_{2} f_{\boldsymbol{Z}}(z) d z$ were null. We now proceed to prove this. Since $\int_{0}^{\pi} \sin ^{p-2} \theta_{1} \cos \theta_{1} d \theta_{1}=0$, it follows that:

$$
\begin{array}{r}
\int_{A} z_{1} f_{Z}(z) d z=\frac{1}{(\sqrt{2 \pi})^{p}} \int_{B} e^{-\frac{1}{2} r^{2}} r^{p} \sin ^{p-2} \theta_{1} \cos \theta_{1} \sin ^{p-3} \theta_{2} \ldots \sin \theta_{p-2} d r d \theta_{1} \ldots d \theta_{p-1} \Rightarrow \\
\int_{A} z_{1} f_{Z}(z) d z=\frac{2 \pi}{(\sqrt{2 \pi})^{p}} J_{p}(\gamma) T_{p-3} \ldots T_{1} \int_{0}^{\pi} \sin ^{p-2} \theta_{1} \cos \theta_{1} d \theta_{1}=0
\end{array}
$$

Also,

$$
\begin{aligned}
& \int_{A} z_{1} z_{2} f_{\mathbf{Z}}(z) d z=\frac{1}{(\sqrt{2 \pi})^{p}} \int_{B} e^{-\frac{1}{2} r^{2}} r^{p+1} \sin ^{p-1} \theta_{1} \cos \theta_{1} \sin ^{p-3} \theta_{2} \cos \theta_{2} \ldots \sin \theta_{p-2} d r d \theta_{1} \ldots d \theta_{p-1} \Rightarrow \\
& \int_{A} z_{1} z_{2} f_{\mathbf{Z}}(z) d z=\frac{2 \pi}{(\sqrt{2 \pi})^{p}} J_{p+1}(\gamma) T_{p-4} \ldots T_{1} \int_{0}^{\pi} \sin ^{p-1} \theta_{1} \cos \theta_{1} d \theta_{1} \int_{0}^{\pi} \sin ^{p-3} \theta_{2} \cos \theta_{2} d \theta_{2}=0
\end{aligned}
$$

By symmetry, $\int_{A} z_{i} f_{\boldsymbol{Z}}(z) d z=\int_{A} z_{1} f_{\boldsymbol{Z}}(z) d z=0$ and $\int_{A} z_{i} z_{j} f_{\boldsymbol{Z}}(z) d z=\int_{A} z_{1} z_{2} f_{\boldsymbol{Z}}(z) d z=$ $0, i \neq j$. 


\section{References}

Chauvet, G., D. Haziza, and E. Lesage. 2017. "Examining Some Aspects of Balanced Sampling in Surveys.” Statistica Sinica 27: 313-334. DOI: http://dx.doi.org/10.5705/ ss.2013.244.

Deville, J.-C. and C.E. Särndal. 1992. "Calibration estimators in survey sampling." Journal of the American Statistical Association 87: 376-382. DOI: http://doi.org/ $10.2307 / 2290268$.

Deville, J.-C. and Y. Tillé. 2004. "Efficient balanced sampling: The cube method." Biometrika 91: 893-912. DOI: http://doi.org/10.1093/biomet/91.4.893.

Deville, J.-C. and Y. Tillé. 2005. "Variance approximation under balanced sampling." Journal of statistical planning and inference 128: 569-591. DOI: https://doi.org/ 10.1016/j.jspi.2003.11.011.

Fuller, W.A. 2009. "Some design properties of a rejective sampling procedure." Biometrika 96: 933-944. DOI: https://doi.org/10.1093/biomet/asp042.

Fuller, W.A., J.C. Legg, and Y. Li. 2017. "Bootstrap variance estimation for rejective sampling." Journal of the American Statistical Association 112: 1562-1570. DOI: https://doi.org/10.1080/01621459.2016.1222285.

Hájek, J. 1964. "Asymptotic theory of rejective sampling with varying probabilities from a finite population.” Ann. Math. Statist. 35: 1491-1523. DOI: https://doi.org/10.1214/ aoms/1177700375.

Hájek, J. 1981. Sampling from a finite population. Statistics: Textbooks and Monographs 37. New York: Marcel Dekker Inc.

Horvitz, D.G. and D.J. Thompson. 1952. "A generalization of sampling without replacement from a finite universe." Journal of the American Statistical Association 47: 663-685. DOI: https://doi.org/10.2307/2280784.

Huang, E.T. and W.A. Fuller. 1978. "Nonnegative regression estimation for sample survey data." Proceedings of the Social Statistics Section, American Statistical Association. Alexandria, VA, 300-305. Available at: https://lib.dr.iastate.edu/rtd/6460 (accessed February 2020).

Legg, J.C. and C.L. Yu. 2010. "A comparison of sample set restriction procedures." Survey Methodology 36: 69-79. Available at: https:/www150.statcan.gc.ca/n1/en/ catalogue/12-001-X201000111249 (accessed February 2020).

Matei, A. and Y. Tillé. 2005. "Evaluation of variance approximations and estimators in maximum entropy sampling with unequal probability and fixed sample size." Journal of Official Statistics 21(4): 543-570. Available at: https://www.scb.se/contentassets/ ca21efb41fee47d293bbee5bf7be7fb3/evaluation-of-variance-approximations-and-estimators-in-maximum-entropy-sampling-with-unequal-probability-and-fixed-samplesize.pdf (accessed February 2020).

Särndal, C.-E., B. Swenson, and J. Wretman. 1992. Model Assisted Survey Sampling. New York: Springer-Verlag.

Received June 2018

Revised March 2019

Accepted June 2019 